\title{
Linear Tearing Mode Stability Equations for a Low Collisionality Toroidal Plasma
}

\author{
J W Connor ${ }^{1}$, R J Hastie ${ }^{1}$ and P Helander ${ }^{2}$ \\ 1 EURATOM/UKAEA Fusion Association, Culham Science Centre, Abingdon, Oxon \\ UK, OX14 3DB \\ 2 Max-Planck-Institut für Plasmaphysik, EURATOM-Association, D-17491, \\ Greifswald, Germany
}

\begin{abstract}
Tearing mode stability is normally analysed using MHD or two-fluid Braginskii plasma models. However for present, or future, large hot tokamaks like JET or ITER the collisionality is such as to place them in the banana regime. Here we develop a linear stability theory for the resonant layer physics appropriate to such a regime. The outcome is a set of 'fluid' equations whose coefficients encapsulate all neoclassical physics: the neoclassical Ohm's law, enhanced ion inertia, cross field transport of particles, heat and momentum all play a role. While earlier treatments have also addressed this type of neoclassical physics we differ in incorporating the more physically relevant 'semi-collisional fluid' regime previously considered in cylindrical geometry; semi-collisional effects tend to screen the resonant surface from the perturbed magnetic field, preventing reconnection. Furthermore we also include thermal physics, which may modify the results. While this electron description is of wide relevance and validity, the fluid treatment of the ions requires the ion banana orbit width to be less than the semi-collisional electron layer. This limits the application of the present theory to low magnetic shear - however this is highly relevant to the sawtooth instability - or to colder ions. The outcome of the calculation is a set of one-dimensional radial differential equations of rather high order. However, various simplifications that reduce the computational task of solving these are discussed. In the collisional regime, when the set reduces to a single second order differential equation, the theory extends previous work by Hahm et al (Phys Fluids 313709 (1988)) to include diamagnetic-type effects arising from plasma gradients, both in Ohm's law and the ion inertia term of the vorticity equation. The more relevant semi-collisional regime pertaining to JET or ITER, is described by a pair of second order differential equations, extending the cylindrical equations of Drake et al. (Phys Fluids 262509 (1983)) to toroidal geometry.
\end{abstract}

\section{Introduction}

Magnetic reconnection is believed to play a role in important tokamak phenomena such as sawteeth, neoclassical tearing modes (NTMs) and disruptions. It is normally analysed using resistive MHD or two-fluid Braginskii plasma models. Present large tokamaks, however, operate in a low collisionality regime where a kinetic model is appropriate. This was recognised in the development of neoclassical tearing mode theory, in both linear [1, 2] and non-linear [3,4] situations, where the perturbed bootstrap current provides an instability source. A related theory of neoclassical bootstrap current driven, twisting parity ballooning modes was presented in Ref. 5. In these theories one can develop a systematic expansion procedure about a resonant surface, $\mathrm{m}=\mathrm{nq}$, where $\mathrm{q}$ is the safety factor, in which effective, fluid-like equations are derived but whose coefficients 
encapsulate kinetic effects, such as particle trapping: 'neoclassical fluid' equations. The linear theory presented in Refs. 2 and 5 was appropriate to a situation where these fluidlike equations were more collisional, in the sense that the collision frequencies $v_{\mathrm{e}, \mathrm{i}}$ exceeded the mode frequency, $\omega$. Thus it omitted 'semi-collisional' electron effects occurring when $\omega \mathrm{v}_{\mathrm{e}} \sim \mathrm{k}_{\|}^{2} \mathrm{v}_{\text {the }}^{2}$, with $\mathrm{k}_{\|}$a parallel wave-number and $\mathrm{v}_{\text {the }}$ the electron thermal velocity, previously considered in cylindrical geometry $[6,7,8,9]$ : in this regime there is a balance between parallel diffusive transport and the mode frequency. These semi-collisional effects tend to screen the resonant surface from the perturbed magnetic field, preventing reconnection and one finds that tearing mode instability requires large $\Delta^{\prime}[8]$, where $\Delta^{\prime}$ is the familiar tearing mode stability parameter.

Reference 8 has given the criterion for the validity of this semi-collisional theory as $\beta_{\mathrm{e}}\left(\mathrm{L}_{\mathrm{s}} / \mathrm{L}_{\mathrm{n}}\right)^{2}>1$, where $\beta_{\mathrm{e}}=2 \mu_{0} \mathrm{n}_{\mathrm{e}} \mathrm{T}_{\mathrm{e}} / \mathrm{B}^{2}$ is the electron beta, $\mathrm{L}_{\mathrm{s}}=\mathrm{Rq} / \hat{\mathrm{s}}$ (where $\hat{\mathrm{s}}=(\mathrm{r} / \mathrm{q})(\mathrm{dq} / \mathrm{dr})$ is the magnetic shear $)$ is the shear length and $\mathrm{L}_{\mathrm{n}}$ is the density scalelength ( $\mathrm{R}$ is the tokamak major radius, a the minor radius and $\mathrm{r}$ the radius of a given flux surface). Large tokamaks such as JET, and eventually ITER, operate in a regime where the collisional limit is not justified. Thus if we take typical ITER parameters $\left[\mathrm{n}_{\mathrm{e}}(\mathrm{r} / \mathrm{a}=0.3) \sim 10^{20} \mathrm{~m}^{-3}, \mathrm{~T}_{\mathrm{e}}(\mathrm{r} / \mathrm{a}=0.3) \sim 22 \mathrm{keV}, \mathrm{B}=5.7 \mathrm{~T}, \mathrm{R} / \mathrm{a}=2.9\right]$, assume $\mathrm{q}=1$ and that this lies near $\mathrm{r} / \mathrm{a} \sim 0.3$, and $\mathrm{L}_{\mathrm{n}} \sim \mathrm{a}$, then $\beta_{\mathrm{e}}\left(\mathrm{L}_{\mathrm{s}} / \mathrm{L}_{\mathrm{n}}\right)^{2} \sim 0.12 / \hat{\mathrm{s}}^{2}$. Clearly for $\hat{\mathrm{s}}<<1$, the semi-collisional regime is appropriate, a situation very relevant to sawtooth modelling.

Here we develop a theory that incorporates these semi-collisional effects into the neoclassical formalism. Furthermore, we include the effects of radial temperature gradients and thermal transport, absent from some earlier treatments (although electron neoclassical transport can be retained, its effect is negligible). The inclusion of thermal effects is of interest because there is evidence, e.g. from T-10 [10], that the radial electron temperature gradient, $\mathrm{dT}_{\mathrm{e}} / \mathrm{dr}$, plays a role in the sawtooth phenomenon, with sawteeth being triggered when it exceeds a critical value. For the narrow semi-collisional resonant layers under discussion here, the question arises of whether to treat the ions as magnetised, $\mathrm{k}_{\perp} \rho_{\mathrm{i}} \leq 1$, or un-magnetised, $\mathrm{k}_{\perp} \rho_{\mathrm{i}} \gg 1$, where $\mathrm{k}_{\perp}$ is a perpendicular wavenumber and $\rho_{\mathrm{i}}$ is the ion Larmor radius. This has only been addressed within a cylindrical model [11] (although the case where the resonant layer is less than $\rho_{\mathrm{s}}$, the ion Larmor radius at the sound speed, has been treated for the cold ion fluid model in toroidal geometry [12]). In this work we consider the magnetised case, which can be justified at low magnetic shear, $\hat{\mathrm{s}}<<1$, a situation again relevant to the sawtooth instability, or for cold ions. The opposite case is extremely challenging, and will be deferred to later work, but suffice it to say that the effects of large ion orbits provide strong stabilisation [11].

In the following sections we develop a set of equations to describe tearing mode stability, namely coupled equations for the perturbed magnetic field, electrostatic potential and electron and ion densities, parallel flows, including the bootstrap current, and temperatures. Thus, Section 2 deals with the solution of the electron gyro-kinetic 
equation and the corresponding ion equation. Section 3 addresses Maxwell's equations and Ohm's law, while Section 4 develops the key vorticity equation. In Section 5 we consider some particular cases of interest particular. Firstly we address the collisional limit, $\omega v_{\mathrm{e}}>>\mathrm{k}_{\|}^{2} \mathrm{v}_{\text {the }}^{2}$, obtaining the generalisation of the results of Ref. 2 to include the effects of temperature gradients. We also obtain the form of the equations for the toroidal version of the semi-collisional mode, appropriate when $\omega v_{\mathrm{e}} \sim \mathrm{k}_{\|}^{2} \mathrm{v}_{\text {the }}^{2}$, generalising the cylindrical results of in Ref. 8 to toroidal geometry. An analytic solution of the stability problem posed by this latter set of equations will be discussed in a later paper. In the final section we discuss the stability problem presented by our general set of equations, their limitations, some possible simplifications and some plausible implications. Some details of the calculations concerning the vorticity equation, including the introduction of the notation of Glasser et al. [13] for toroidal geometry, appear in Appendix A. For convenience, Appendix B collects together some of the many symbols and notation introduced in the text.

\section{The Gyro-kinetic Equations}

\section{(i) General Discussion}

The gyro-kinetic equation for species $\mathrm{j}$ is $[5,14]$

$$
\left(\mathrm{v}_{\|} \mathbf{b}+\mathbf{v}_{\mathrm{dj}}\right) \cdot \nabla \mathrm{g}_{\mathrm{j}}-\mathrm{i} \omega \mathrm{g}_{\mathrm{j}}-\mathrm{C}_{\mathrm{j}}\left(\mathrm{g}_{\mathrm{j}}\right)=-\mathrm{i} \frac{\mathrm{e}_{\mathrm{j}}}{\mathrm{T}_{\mathrm{j}}} \mathrm{f}_{0 \mathrm{j}}\left(\omega-\omega_{* \mathrm{j}}^{\mathrm{T}}\right)\left[\mathrm{J}_{0}\left(\mathrm{z}_{\mathrm{j}}\right)\left(\Phi-\mathrm{v}_{\|} \mathrm{A}_{\|}\right)+\frac{\mathrm{v}_{\perp}}{\mathrm{k}_{\perp}} \tilde{\mathrm{B}}_{\|} \mathrm{J}_{1}\left(\mathrm{z}_{\mathrm{j}}\right)\right]
$$

where $\mathbf{b}$ is a unit vector along the magnetic field, $v_{\|}$is the particle velocity along the magnetic field, $\Phi$ is the perturbed electrostatic potential, $A_{\|}$is the perturbed parallel component of the vector potential, $\hat{\mathrm{B}}_{\|}$is the perturbed parallel magnetic field and we have written the perturbed distribution as

$$
\delta f_{j}=-\frac{e_{j} \Phi}{T_{j}} f_{0 j}+g_{j} e^{i L_{j}}
$$

Here $L_{j}=\left|\mathbf{k} \times \mathbf{v}_{\perp} / \Omega_{\mathrm{j}}\right|$, with $\mathrm{v}_{\perp}$ the velocity perpendicular to the magnetic field, is the gyro-phase factor, $\mathrm{J}_{0,1}$ are Bessel functions of argument $\mathrm{z}_{\mathrm{j}}=\mathrm{k}_{\perp} \mathrm{v}_{\perp} / \Omega_{\mathrm{j}}, \mathrm{f}_{0 \mathrm{j}}$ are Maxwellian distributions and

$$
\omega_{*_{j}}^{T}=\omega_{* j}\left[1+\eta_{j}\left(u_{j}^{2}-\frac{3}{2}\right)\right], u_{j}^{2}=\frac{m_{j} v^{2}}{2 T_{j}}, \omega_{*_{j}}=-n \frac{T_{j}}{e_{j}} \frac{d \ln n_{j}}{\partial \psi}
$$




$$
\mathbf{v}_{\mathrm{dj}}=\frac{\mathbf{b}}{\Omega_{\mathrm{j}}} \times\left(\frac{\mathrm{v}_{\perp}^{2}}{2} \nabla \ln \mathrm{B}+\mathrm{v}_{\|}^{2} \mathbf{\kappa}\right), \quad \Omega_{\mathrm{j}}=\frac{\mathrm{e}_{\mathrm{j}} \mathrm{B}}{\mathrm{m}_{\mathrm{j}}}, \quad \boldsymbol{\kappa}=\mathbf{b} . \nabla \mathbf{b}
$$

with $\mathrm{v}$ the particle speed, $\Omega_{\mathrm{j}}$ the cyclotron frequency of species $\mathrm{j}, \boldsymbol{\kappa}$ is the curvature vector, $\mathrm{n}$ the toroidal mode number and all gradients are taken at constant $(\mu, \mathrm{v})$ with $\mu$ the magnetic moment, or $(\lambda \mathrm{v})$ with $\lambda=2 \mu / \mathrm{v}^{2}$ (thus $\mathrm{v}_{\|}=\sigma \mathrm{v} \sqrt{1-\lambda \mathrm{B}}$, with $\sigma=\operatorname{sign}\left(\mathrm{v}_{\|}\right)$). If $\theta$ is defined so that the safety factor, $\mathrm{q}=\mathbf{B} \cdot \nabla \varphi / \mathbf{B} \cdot \nabla \theta$, is a flux function, then

$$
\nabla_{\|}=\mathbf{b} \cdot \nabla \theta \frac{\partial}{\partial \theta}+\mathbf{b} \cdot \nabla \varphi \frac{\partial}{\partial \varphi}=\frac{\mathrm{I}}{\mathrm{R}^{2} \mathrm{~B}}\left(\frac{\partial}{\partial \varphi}+\frac{1}{\mathrm{q}} \frac{\partial}{\partial \theta}\right)
$$

with $I=R B_{\varphi}$, so if we write $g_{j}$ as

$$
g_{j}(r, \theta, \varphi)=\hat{g}_{j}(r, \theta) e^{i(n \varphi-m \theta)}
$$

then

$$
\nabla_{\|} g_{j}=\frac{I}{R^{2} B q}\left[i(n q-m) \hat{g}_{j}+\frac{\partial \hat{g}_{j}}{\partial \theta}\right] e^{i(n \varphi-m \theta)}
$$

where

$$
\mathrm{nq}-\mathrm{m} \simeq \mathrm{nq} \mathrm{q}^{\prime}, \mathrm{q}^{\prime}=\frac{\mathrm{dq}}{\mathrm{d} \psi}
$$

with $\mathrm{x}=\psi-\psi_{\mathrm{s}}, \psi_{\mathrm{s}}$ being the resonant surface where $\mathrm{m}=\mathrm{nq}$, and prime denotes a derivative with respect to $\mathrm{x}$.. It is convenient later to introduce

$$
\hat{\nabla}_{\|}=\frac{\mathrm{I}}{\mathrm{R}^{2} \mathrm{~Bq}} \frac{\partial}{\partial \theta}
$$

Dropping carets we obtain

$$
\begin{aligned}
& \frac{\mathrm{Iv}_{\|}}{\mathrm{R}^{2} \mathrm{~Bq}}\left(\frac{\partial \mathrm{g}_{\mathrm{j}}}{\partial \theta}+\mathrm{inq}^{\prime} \mathrm{xg}_{\mathrm{j}}\right)+\mathbf{v}_{\mathrm{dj}} \cdot \nabla \mathrm{g}_{\mathrm{j}}-\mathrm{i} \omega \mathrm{g}_{\mathrm{j}}-\mathrm{C}_{\mathrm{j}}\left(\mathrm{g}_{\mathrm{j}}\right) \\
& =-\frac{\mathrm{ie}_{\mathrm{j}}}{\mathrm{T}_{\mathrm{j}}} \mathrm{f}_{0 \mathrm{j}}\left(\omega-\omega_{* \mathrm{j}}^{\mathrm{T}}\right)\left[\mathrm{J}_{0}\left(\Phi-\mathrm{v}_{\|} \mathrm{A}_{\|}\right)+\mathrm{J}_{1} \frac{\mathrm{v}_{\perp} \tilde{\mathrm{B}}_{\|}}{\mathrm{k}_{\perp}}\right]
\end{aligned}
$$


which we rewrite by expanding the Bessel functions for small $\mathrm{z}_{\mathrm{j}}$ and by introducing $\mathrm{h}_{\mathrm{j}}$ :

$$
g_{j}=\frac{e_{j}}{T_{j}}\left(1-\frac{\omega_{* j}^{T}}{\omega}\right) \Psi f_{0 j}+h_{j}
$$

where

$$
\mathrm{A}_{\|}=\nabla_{\|} \Psi / \mathrm{i} \omega
$$

Thus the parallel electric field is given by

$$
\mathrm{E}_{\|}=-\frac{\mathrm{I}}{\mathrm{R}^{2} \mathrm{~Bq}}\left(\frac{\partial}{\partial \theta}+\text { inq' }^{\prime}\right)(\Phi-\Psi)
$$

Then the fundamental kinetic equations are

$$
\begin{aligned}
& \frac{\mathrm{Iv}_{\|}}{\mathrm{R}^{2} \mathrm{~Bq}}\left(\frac{\partial \mathrm{h}_{\mathrm{j}}}{\partial \theta}+\mathrm{inq}^{\prime} \mathrm{xh}_{\mathrm{j}}\right)+\mathrm{v}_{\mathrm{dj}} \cdot \nabla \mathrm{h}_{\mathrm{j}}-\mathrm{i} \omega \mathrm{h}_{\mathrm{j}}-\mathrm{C}_{\mathrm{j}}\left(\mathrm{h}_{\mathrm{j}}\right) \\
& =-\frac{\mathrm{ie}_{\mathrm{j}}}{\mathrm{T}_{\mathrm{j}}} \mathrm{f}_{0 \mathrm{j}}\left(\omega-\omega_{* \mathrm{j}}^{\mathrm{T}}\right)\left(\Phi-\Psi+\frac{\mathrm{v}_{\perp}{ }^{2} \tilde{\mathrm{B}}_{\|}}{2 \Omega_{\mathrm{j}}}\right)+\frac{\mathrm{e}_{\mathrm{j}}}{\mathrm{T}_{\mathrm{j}}}\left(1-\frac{\omega_{* \mathrm{j}}^{\mathrm{T}}}{\omega}\right) \mathrm{f}_{0 \mathrm{j}} \mathbf{v}_{\mathrm{dj}} \cdot \nabla \Psi
\end{aligned}
$$

We will solve these equations by introducing appropriate ordering schemes for electrons and ions. It is convenient to introduce an ordering parameter $\varepsilon$, where $\varepsilon=\left(\rho_{\mathrm{e}} / \delta\right)^{\lambda}$ with $\delta$ the resonant layer width (where semi-collisional effects are manifest) and $\lambda$ an exponent to be determined below, and $\left(\mathrm{m}_{\mathrm{e}} / \mathrm{m}_{\mathrm{i}}\right)^{1 / 2}$, defining $\left(\mathrm{m}_{\mathrm{e}} / \mathrm{m}_{\mathrm{i}}\right)^{1 / 2}=\varepsilon^{\mu}$. We can then exhibit the ordering of terms on the left-hand side of eqn. (13):

$$
\begin{array}{lcccccc} 
& \omega_{\mathrm{bj}} & \mathrm{k}_{\|} \mathrm{V}_{\text {thj }} & \omega_{\mathrm{drj}} & \omega & v_{\mathrm{j}} & \mathrm{k}_{\mathrm{r}}^{2} \mathrm{D}_{\perp \mathrm{j}}^{\text {neo }} \\
\mathrm{j}=\text { electrons } & 1 & \varepsilon^{\lambda+1} & \varepsilon^{\lambda} & \varepsilon^{\lambda+2} & \varepsilon^{\lambda} & \varepsilon^{3 \lambda} \\
\mathrm{j}=\text { ions } & 1 & \varepsilon^{\lambda+1} & \varepsilon^{\lambda-\mu} & \varepsilon^{\lambda+2-\mu} & \varepsilon^{\lambda} & \varepsilon^{3 \lambda-2 \mu}
\end{array}
$$

Here $\omega_{b j}$ is the bounce/transit frequency, $\mathrm{k}_{\|} \mathrm{v}_{\text {thj }}$ the transit frequency over a wavelength $\mathrm{Rq} / \delta \mathrm{q}^{\prime}$ associated with the resonant layer width, $\delta, \omega_{\mathrm{drj}}$ the radial magnetic drift frequency, respectively, for species j. (Although not explicit in eqn. (13), we have 
also included $\mathrm{k}_{\mathrm{r}}^{2} \mathrm{D}_{\perp \mathrm{j}}^{\mathrm{neo}}$, the neoclassical collisional radial diffusion rate associated with species $\mathrm{j}$, which appears during the expansion of eqn. (13) and enters our final macroscopic equations.) This ordering automatically fulfils the semi-collisional condition, $\omega v_{\mathrm{e}} \sim \mathrm{k}_{\|}^{2} \mathrm{v}_{\text {the }}^{2}$. However, there are a number of constraints on the parameters $\lambda$ and $\mu$ that one may wish to impose:

- To develop a systematic and convenient $\varepsilon$-expansion for ions: $\lambda>\mu$

- To neglect ion sound: $\mathrm{k}_{\|} \mathrm{v}_{\text {thi }} / \omega \leq 1 \Rightarrow \mu \geq 1$

- To neglect cross-field ion neoclassical transport: $v_{\mathrm{i}} \rho_{\mathrm{i}}{ }^{2} / \omega \delta^{2} \leq 1 \Rightarrow \lambda \geq 1+\mu / 2$ (equality implies retention)

These criteria are displayed in Fig. 1

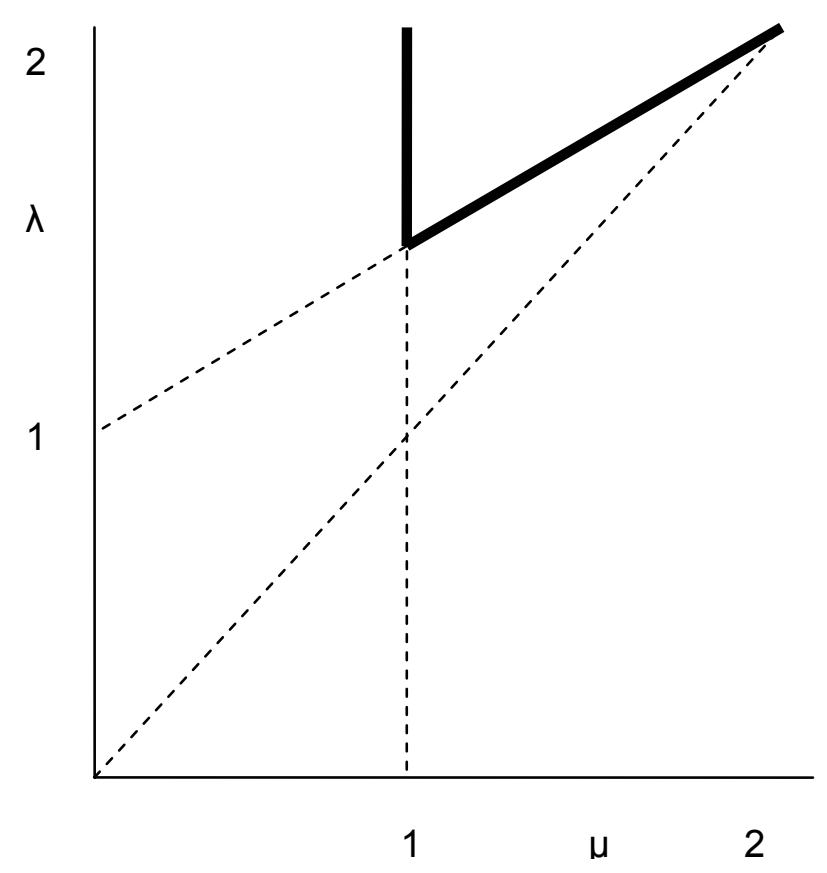

Fig. 1: The 'operating space' for choosing the exponents $\lambda$ and $\mu$; $\lambda$ is involved in the exponents of the powers of $\varepsilon=\left(\rho_{\mathrm{e}} / \delta\right)^{\lambda}$, representing the orders of the terms of the gyro-kinetic equations as shown in eqn. (14), while $\left(\mathrm{m}_{\mathrm{e}} / \mathrm{m}_{\mathrm{i}}\right)^{1 / 2}=\varepsilon^{\mu}$.

Thus we could choose $\lambda=2, \mu=3 / 2$; this allows us to ignore ion neoclassical transport and ion sound effects. Alternatively we can choose $\lambda=7 / 4, \mu=3 / 2$ which allows us to retain ion neoclassical transport. Having satisfied ourselves that there is a self-consistent ordering scheme for an expansion that allows us to ignore ion sound effects and retain ion 
neoclassical transport we will proceed with this by including the various physical effects at appropriate stages in the expansion, rather than employing the specific but complicated choice above, which would lead to many 'empty orders' in a formal expansion.

We will find that the solution of eqn. (13), order by order, mirrors that in standard neoclassical theory, with arbitrary functions being determined by collisional constraints arising from periodicity of the bounce/transit motion. For these constraints we use momentum conserving, pitch-angle scattering collision models. The lowest order solution for $h_{j}$ is Maxwellian with perturbed densities, $\hat{n}_{j}$, and temperatures, $\hat{T}_{j}$, constant on a flux surface. Next order determines perturbed parallel flows from which one can compute the ion flow and bootstrap current driven by the perturbed gradients. Finally, solubility conditions in third order lead to 'neoclassical fluid equations' for $\hat{n}_{j}$ and $\hat{T}_{j}$ in response to the electromagnetic perturbations, which describe cross-field neoclassical transport, parallel collisional electron transport, neoclassical compressibility and the mode frequency, $\omega$. However one can reasonably ignore the small electron cross-field transport to obtain closed algebraic expressions for the semi-collisional perturbed electron density and temperature.

\section{(ii) Electron Solution}

Dropping the e suffix on $\mathrm{h}_{\mathrm{e}}$ for brevity and introducing the proton charge $\mathrm{e}$, so that $\mathrm{e}_{\mathrm{j}}=-\mathrm{e}$ for electrons, we have

$$
\begin{aligned}
& \frac{\mathrm{Iv}_{\|}}{\mathrm{R}^{2} \mathrm{~Bq}}\left(\frac{\partial \mathrm{h}}{\partial \theta}+\text { inq'xh }\right)+\mathbf{v}_{\mathrm{de}} \cdot \nabla \mathrm{h}-\mathrm{i} \omega \mathrm{h}-\mathrm{C}_{\mathrm{e}}(\mathrm{h}) \\
& =\frac{\mathrm{ie}}{\mathrm{T}_{\mathrm{e}}} \mathrm{f}_{0 \mathrm{e}}\left(\omega-\omega_{*_{\mathrm{e}}^{\mathrm{T}}}^{\mathrm{T}}\right)\left(\Phi-\Psi+\frac{\mathrm{v}_{\perp}{ }^{2} \tilde{\mathrm{B}}_{\|}}{2 \Omega_{\mathrm{e}}}\right)+\frac{\mathrm{e}}{\mathrm{T}_{\mathrm{e}}}\left(1-\frac{\omega_{*_{\mathrm{e}}}^{\mathrm{T}}}{\omega}\right) \mathrm{f}_{0 \mathrm{e}} \mathbf{v}_{\mathrm{de}} \cdot \nabla \Psi
\end{aligned}
$$

We order the various terms in eqn. (15) such that in zeroth order

$$
\frac{\partial \mathrm{h}_{0}}{\partial \theta}=0 \Rightarrow \mathrm{h}_{0}=\mathrm{h}_{0}(\mathrm{v}, \lambda, \mathrm{x}, \sigma)
$$

and in first order

$$
\frac{\mathrm{Iv}_{\|}}{\mathrm{R}^{2} \mathrm{~Bq}} \frac{\partial \mathrm{h}_{1}}{\partial \theta}=\mathrm{C}_{\mathrm{e}}\left(\mathrm{h}_{0}\right)-\mathrm{v}_{\mathrm{dre}} \frac{\partial \mathrm{h}_{0}}{\partial \mathrm{x}}+\frac{\mathrm{e}}{\mathrm{T}_{\mathrm{e}}}\left(1-\frac{\omega_{*_{\mathrm{e}}}^{\mathrm{T}}}{\omega}\right) \mathrm{f}_{0 \mathrm{e}} \mathrm{v}_{\mathrm{dre}} \frac{\partial \Psi}{\partial \mathrm{x}}
$$

In eqn. (13) 


$$
\mathrm{v}_{\mathrm{drj}}=\mathbf{v}_{\mathrm{dj}} \cdot \nabla \psi=\frac{\mathrm{Iv}_{\|}}{\mathrm{R}^{2} \mathrm{~Bq}} \frac{\partial}{\partial \theta}\left(\frac{\mathrm{Iv}_{\|}}{\Omega_{\mathrm{j}}}\right)
$$

and everything but the collision operator is annihilated by an orbit average. For passing particles we annihilate by applying the operator $\left\langle\mathrm{B}(\ldots) / \mathrm{v}_{\|}\right\rangle$, where

$$
\langle\ldots\rangle=\oint \frac{(\ldots) \mathrm{d} \theta}{\mathbf{B} . \nabla \theta} / \oint \frac{\mathrm{d} \theta}{\mathbf{B} . \nabla \theta}=\oint(\ldots) \mathrm{R}^{2} \mathrm{~d} \theta / \oint \mathrm{R}^{2} \mathrm{~d} \theta
$$

since

$$
\frac{1}{\text { B. } \nabla \theta}=\frac{\mathrm{q}}{\text { B. } \nabla \varphi}=\frac{\mathrm{qR}^{2}}{\mathrm{I}}
$$

For trapped particles we integrate along the bounce orbit, summing over $\sigma$ in the usual way [15] to obtain the constraint:

$$
\left\langle\frac{\mathrm{B}}{\mathrm{v}_{\|}} \mathrm{C}_{\mathrm{e}}\left(\mathrm{h}_{0}\right)\right\rangle=0
$$

which determines $\mathrm{h}_{0}$, yielding

$$
\mathrm{h}_{0}=\left[\frac{\hat{\mathrm{n}}_{\mathrm{e}}}{\mathrm{n}_{\mathrm{e}}}+\left(\mathrm{u}^{2}-\frac{3}{2}\right) \frac{\hat{\mathrm{T}}_{\mathrm{e}}}{\mathrm{T}_{\mathrm{e}}}\right] \mathrm{f}_{0 \mathrm{e}}
$$

It should be stressed that $\hat{n}_{e}$ and $\hat{T}_{e}$ (and later the same quantities for ions) only represent the contributions to perturbed density and temperature from $h_{e}$, whereas in calculating the full quantities, $\tilde{n}_{e}$ and $\tilde{T}_{e}$ one must recall we have represented $f_{e}$ in terms of first $g_{e}$ and then $h_{e}$. One can now integrate eqn. (17) to obtain

$$
\mathrm{h}_{1}=\frac{\mathrm{Iv}_{\|}}{\Omega_{\mathrm{e}}}\left[\frac{\mathrm{e}}{\mathrm{T}_{\mathrm{e}}}\left(1-\frac{\omega_{* \mathrm{e}}^{\mathrm{T}}}{\omega}\right) \Psi^{\prime} \mathrm{f}_{0 \mathrm{e}}-\mathrm{h}_{0}^{\prime}\right]+\overline{\mathrm{h}}_{1}(\mathrm{v}, \lambda, \mathrm{x}, \sigma)
$$

The next order equation is

$$
\mathrm{v}_{\|} \hat{\nabla}_{\|} \mathrm{h}_{2}+\mathrm{v}_{\|} \hat{\nabla}_{\|}\left(\frac{\mathrm{Iv}_{\|}}{\Omega_{\mathrm{e}}}\right) \frac{\partial \mathrm{h}_{1}}{\partial \mathrm{x}}+\frac{\mathrm{iv}_{\|} \mathrm{Inq}^{\prime} \mathrm{x}}{\mathrm{qR}^{2} \mathrm{~B}} \mathrm{~h}_{0}=\mathrm{C}_{\mathrm{e}}\left(\mathrm{h}_{1}\right)
$$

Applying the operation used in eqn. (21), we obtain the constraint 


$$
\left\langle\frac{\mathrm{B}}{\mathrm{v}_{\|}} \mathrm{C}_{\mathrm{e}}\left(\mathrm{h}_{1}\right)\right\rangle=\frac{\mathrm{iInq}^{\prime} \mathrm{x}}{\mathrm{q}} \quad\left\langle\frac{1}{\mathrm{R}^{2}}\right\rangle \mathrm{h}_{0}
$$

which determines $\overline{\mathrm{h}}_{1}(\mathrm{v}, \lambda, \mathrm{x}, \sigma)$.

We introduce a momentum conserving, pitch angle scattering electron collision operator:

$$
\begin{gathered}
C_{e}(h)=v_{e i}(v)\left[L h+\frac{m_{e}}{T_{e}} v_{\|} u_{\| i} f_{0 e}\right]+v_{e e}(v)\left[L h+\frac{m_{e}}{T_{e}} v_{\|} u_{\| e}^{*} f_{0 e}^{*}\right] \\
u_{\| i}=\left(1 / n_{i}\right) \int d^{3} v v_{\|} h_{i} d^{3} v, u_{\| e}^{*}=\int d^{3} v v_{e e}(v) v_{\|} h_{1 e} / \int d^{3} v\left(m_{e} v_{\|}^{2} / T_{e}\right) v_{e e} f_{0 e} \\
v_{e i}=v_{0} /\left(m_{e} v^{2} / 2 T_{e}\right)^{3 / 2} ; v_{e e}=v_{0} \phi\left(m_{e} v^{2} / 2 T_{e}\right) /\left(m_{e} v^{2} / 2 T_{e}\right)^{3 / 2} \\
v_{0}=\frac{\sqrt{2} \pi n_{e} e^{4} \ln \Lambda}{m_{e}^{1 / 2} T_{e}^{3 / 2}}
\end{gathered}
$$

where the Lorentz collision operator, L, is given by

$$
\mathrm{L} \equiv \frac{2 \mathrm{v}_{\|}}{\mathrm{v}^{2} \mathrm{~B}} \frac{\partial}{\partial \lambda} \lambda \mathrm{v}_{\|} \frac{\partial}{\partial \lambda}
$$

and

$$
\phi(x)=(1-1 / 2 x) \eta(x)+\eta^{\prime}(x), \eta(x)=(2 / \sqrt{\pi}) \int_{0}^{\infty} e^{-t} t^{1 / 2} d t, \eta^{\prime}(x)=d \eta / d x
$$

Then we obtain an equation for $\overline{\mathrm{h}}_{1}$ :

$$
\begin{aligned}
-\frac{\mathrm{IB}}{\Omega_{\mathrm{e}}}\left[\frac{\mathrm{e}}{\mathrm{T}_{\mathrm{e}}}\right. & \left.\left(1-\frac{\omega_{*}^{\mathrm{T}}}{\omega}\right) \Psi^{\prime} \mathrm{f}_{0 \mathrm{e}}-\mathrm{h}_{0}^{\prime}\right]+\frac{2}{\mathrm{v}^{2}} \frac{\partial}{\partial \lambda} \lambda\left\langle\mathrm{v}_{\|}\right\rangle \frac{\partial \overline{\mathrm{h}}_{1}}{\partial \lambda} \\
= & \frac{\mathrm{iInq^{ \prime } \mathrm { x }}}{\mathrm{q} v_{\mathrm{ei}}(\mathrm{v})}\left\langle\frac{1}{\mathrm{R}^{2}}\right\rangle \mathrm{h}_{0}-\frac{\mathrm{m}_{\mathrm{e}}}{\mathrm{T}_{\mathrm{e}}} \mathrm{f}_{0 \mathrm{e}}\left[\frac{v_{\mathrm{ei}}}{v_{\mathrm{e}}}\left\langle\mathrm{Bu}_{\| \mathrm{i}}\right\rangle+\frac{v_{\mathrm{ee}}}{v_{\mathrm{e}}}\left\langle\mathrm{Bu}_{\| \mathrm{e}}^{*}\right\rangle\right]
\end{aligned}
$$

with $v_{\mathrm{e}}(\mathrm{v})=v_{\mathrm{ee}}(\mathrm{v})+v_{\mathrm{ei}}(\mathrm{v})$, so that

$$
\begin{aligned}
& \mathrm{h}_{1}=\frac{\mathrm{I}}{\Omega_{\mathrm{e}}}\left[\mathrm{v}_{\|}-\frac{\mathrm{B}}{\mathrm{B}_{0}} \hat{\mathrm{V}}_{\|}\right]\left[\frac{\mathrm{e} \Psi^{\prime}}{\mathrm{T}_{\mathrm{e}}}\left(1-\frac{\omega_{\mathrm{*}_{\mathrm{e}}}^{\mathrm{T}}}{\omega}\right) \mathrm{f}_{0 \mathrm{e}}-\mathrm{h}_{0}^{\prime}\right] \\
& -\mathrm{i} \frac{\mathrm{Inq} \mathrm{x}^{\prime} \mathrm{x}}{\mathrm{q} v_{\mathrm{e}}}\left\langle\frac{1}{\mathrm{R}^{2}}\right\rangle \frac{\hat{\mathrm{V}}_{\|}}{\mathrm{B}_{0}} \mathrm{~h}_{0}+\frac{\mathrm{m}_{\mathrm{e}}}{\mathrm{T}_{\mathrm{e}}} \frac{\hat{\mathrm{V}}_{\|}}{\mathrm{B}_{0}} \mathrm{f}_{0 \mathrm{e}}\left[\frac{v_{\mathrm{ei}}}{v_{\mathrm{e}}}\left\langle\mathrm{Bu}_{\| \mathrm{i}}\right\rangle+\frac{v_{\mathrm{ee}}}{v_{\mathrm{e}}}\left\langle\mathrm{Bu}_{\| \mathrm{e}}^{*}\right\rangle\right]
\end{aligned}
$$


where

$$
\hat{\mathrm{V}}_{\|}(\mathrm{v}, \lambda, \psi, \sigma)=\frac{\sigma \mathrm{v}^{2} \mathrm{~B}_{0}}{2} \int_{\lambda}^{\lambda_{\mathrm{c}}} \frac{\mathrm{d} \lambda^{\prime}}{\left\langle\left|\mathrm{v}_{\|}\left(\lambda^{\prime}\right)\right|\right\rangle}
$$

in which $\mathrm{B}_{0}$ is arbitrary (for later convenience we let $\mathrm{B}_{0}=\left\langle\mathrm{B}^{2}\right\rangle^{1 / 2}$ ) and $\lambda_{\mathrm{c}}=1 / \mathrm{B}_{\max }$.

We can use eqn. (30) to calculate $\mathrm{u}_{\| \mathrm{e}}=\int \mathrm{d}^{3} \mathrm{v} \mathrm{v}_{\|} \mathrm{h}_{1 \mathrm{e}} / \mathrm{n}_{\mathrm{e}}$, where $\int d^{3} v=\sum_{\sigma} B \int \pi v^{3} d v d \lambda /\left|v_{\|}\right|$, in order to help determine $j_{\|}$later:

$$
\begin{aligned}
& u_{\| e}=\frac{B}{\left\langle B^{2}\right\rangle} \frac{f_{c}}{\left(1-0.37 f_{c}\right)}\left(0.57+0.06 f_{c}\right)\left\langle B u_{\| i}\right\rangle+\frac{I}{\Omega_{e}} \frac{T_{e}}{m_{e}}\left[\frac{e \Psi^{\prime}}{T_{e}}\left(1-\frac{\omega_{* e}}{\omega}\left(1+\eta_{e}\right)\right)-\frac{\hat{n}_{e}^{\prime}}{n_{e}}-\frac{\hat{T}_{e}^{\prime}}{T_{e}}\right] \\
& -\frac{\mathrm{I}}{\Omega_{\mathrm{e}}} \frac{\mathrm{T}_{\mathrm{e}}}{\mathrm{m}_{\mathrm{e}}} \frac{\mathrm{B}^{2}}{\left\langle\mathrm{~B}^{2}\right\rangle} \frac{\mathrm{f}_{\mathrm{c}}}{\left(1-0.37 \mathrm{f}_{\mathrm{c}}\right)}\left\{\left[\frac{\mathrm{e} \Psi^{\prime}}{\mathrm{T}_{\mathrm{e}}}\left(1-\frac{\omega_{*_{\mathrm{e}}}}{\omega}\right)-\frac{\hat{\mathrm{n}}_{\mathrm{e}}^{\prime}}{\mathrm{n}_{\mathrm{e}}}\right]\left(0.57+0.06 \mathrm{f}_{\mathrm{c}}\right)-\left[\frac{\mathrm{e} \Psi^{\prime}}{\mathrm{T}_{\mathrm{e}}} \frac{\omega_{*_{\mathrm{e}}}}{\omega} \eta_{\mathrm{e}}+\frac{\hat{\mathrm{T}}_{\mathrm{e}}^{\prime}}{\mathrm{T}_{\mathrm{e}}}\right]\left(1.07-0.44 \mathrm{f}_{\mathrm{c}}\right)\right\} \\
& -\frac{i \operatorname{Inq} q^{\prime} x}{q}\left\langle\frac{1}{R^{2}}\right\rangle \frac{B}{\left\langle B^{2}\right\rangle} \frac{f_{c}}{\left(1-0.37 f_{c}\right)} \frac{T_{e}}{m_{e}} \tau_{e i}\left[\left(1.84-0.33 f_{c}\right) \frac{\hat{n}_{e}}{n_{e}}+\left(4.46-1.26 f_{c}\right) \frac{\hat{T}_{e}}{T_{e}}\right]
\end{aligned}
$$

Here the circulating fraction of particles is given by $\mathrm{f}_{\mathrm{c}}=\frac{3 \mathrm{~B}_{0}^{2}}{4} \int_{0}^{\lambda_{\mathrm{c}}} \frac{\lambda \mathrm{d} \lambda}{\langle\sqrt{1-\lambda \mathrm{B}}\rangle}$ and $\tau_{\mathrm{ei}}=3 \sqrt{\pi} / 4 v_{0}$ is the electron-ion momentum exchange time.

The equation for $\mathrm{h}_{3}$ appears in next order:

$$
\begin{aligned}
& \mathrm{v}_{\|} \hat{\nabla}_{\|} \mathrm{h}_{3}+\mathrm{v}_{\|} \hat{\nabla}_{\|}\left(\frac{\mathrm{Iv}_{\|}}{\Omega_{\mathrm{e}}}\right) \frac{\partial \mathrm{h}_{2}}{\partial \psi}+\frac{\mathrm{iInq}^{\prime} \mathrm{x}}{\mathrm{qR}^{2} \mathrm{~B}} \mathrm{v}_{\|} \mathrm{h}_{1}-\mathrm{i} \omega \mathrm{h}_{0} \\
= & \mathrm{C}_{\mathrm{e}}\left(\mathrm{h}_{2}\right)+\frac{\mathrm{ie}}{\mathrm{T}_{\mathrm{e}}} \mathrm{f}_{0 \mathrm{e}}\left(\omega-\omega_{*_{\mathrm{e}}}^{\mathrm{T}}\right)\left(\Phi-\Psi+\frac{\mathrm{v}_{\perp}{ }^{2} \tilde{\mathrm{B}}_{\|}}{2 \Omega_{\mathrm{e}}}\right)
\end{aligned}
$$

to which we apply the operator

$$
\left\langle\int(\ldots) \mathrm{d}^{3} \mathrm{v}\right\rangle=\sum_{\sigma}\left\langle\mathrm{B} \int(\ldots) \frac{\pi \mathrm{v}^{3} \mathrm{dvd} \lambda}{\left|\mathrm{v}_{\|}\right|}\right\rangle
$$


that annihilates the first terms on both the left and the right. We evaluate the other terms obtaining a first equation for $\hat{n}_{e}$ and $\hat{\mathrm{T}}_{\mathrm{e}}$ (the details can be found in Ref. 16). For completeness we retain the small neoclassical cross-field transport terms. These arise from the annihilation of the $\mathrm{h}_{2}$ term in eqn. (33), using eqn. (24) for $\mathrm{h}_{2}$; it is this interaction of collisions and magnetic drifts that gives rise to neoclassical effects. This is a lengthy calculation and is recorded in Ref. 16, where it is performed for the simpler Lorentz collision model as an illustration. These terms are negligibly small and will henceforth be ignored in this paper. Furthermore we ignore small terms, $\sim\left(\mathrm{k}_{\|} \mathrm{u}_{\| \mathrm{i}} / \omega\right)$, which is justified later by the calculation of $u_{\| i}$ in eqn. (49). The final result is:

$$
\begin{aligned}
& i \omega \frac{\hat{n}_{\mathrm{e}}}{\mathrm{n}_{\mathrm{e}}}+\frac{\mathrm{ie}}{\mathrm{T}_{\mathrm{e}}}\langle\Phi-\Psi\rangle\left(\omega-\omega_{*_{\mathrm{e}}}\right)-\mathrm{i}\left\langle\frac{\tilde{\mathrm{B}}_{\|}}{\mathrm{B}}\right\rangle\left[\omega-\omega_{*_{\mathrm{e}}}\left(1+\eta_{\mathrm{e}}\right)\right] \\
& -\mathrm{D}_{\perp \mathrm{e}}^{\mathrm{neo}}\left[\frac{\mathrm{e} \Psi^{\prime \prime}}{\mathrm{T}_{\mathrm{e}}}\left(1-\frac{\omega_{*}}{\omega}\left(1-\frac{\eta_{\mathrm{e}}}{2}\right)\right)-\frac{\hat{\mathrm{n}}^{\prime \prime}}{\mathrm{n}_{\mathrm{e}}}+\frac{\hat{\mathrm{T}}^{\prime \prime}}{2 \mathrm{~T}_{\mathrm{e}}}\right] \\
& =\left(\frac{\mathrm{Inq} \mathrm{q}^{\prime} \mathrm{x}}{\mathrm{q}\left\langle\mathrm{B}^{2}\right\rangle^{1 / 2}}\left\langle\frac{1}{\mathrm{R}^{2}}\right\rangle\right)^{2} \frac{\mathrm{f}_{\mathrm{c}}}{\left(1-0.37 \mathrm{f}_{\mathrm{c}}\right)} \frac{\mathrm{T}_{\mathrm{e}}}{\mathrm{m}_{\mathrm{e}} v_{0}}\left[\left(2.44-0.45 \mathrm{f}_{\mathrm{c}}\right) \frac{\hat{\mathrm{n}}_{\mathrm{e}}}{\mathrm{n}_{\mathrm{e}}}+\left(5.94-1.69 \mathrm{f}_{\mathrm{c}}\right) \frac{\hat{\mathrm{T}}_{\mathrm{e}}}{\mathrm{T}_{\mathrm{e}}}\right]
\end{aligned}
$$

where, for the Lorentz collision model, $D_{\perp e}^{\text {neo }}=\frac{T_{e} I^{2}}{m_{e} \Omega_{0}^{2} \tau_{e i}}\left\langle\frac{B_{0}^{2}}{B^{2}}-f_{c}\right\rangle$ is the neoclassical cross-field particle diffusivity. A similar equation for the electron energy balance can be derived by applying the operation

$$
\left\langle\int(\ldots) \frac{\mathrm{m}_{\mathrm{e}} \mathrm{v}^{2}}{2} \mathrm{~d}^{3} \mathrm{v}\right\rangle
$$

to eqn. (33) to obtain:

$$
\begin{aligned}
& \frac{3}{2} \mathrm{i} \omega\left(\frac{\hat{n}_{\mathrm{e}}}{\mathrm{n}_{\mathrm{e}}}+\frac{\hat{\mathrm{T}}_{\mathrm{e}}}{\mathrm{T}_{\mathrm{e}}}\right)+\frac{3}{2} \mathrm{i} \frac{\mathrm{e}}{\mathrm{T}_{\mathrm{e}}}\langle\Phi-\Psi\rangle\left(\omega-\omega_{*_{\mathrm{e}}}\left(1+\eta_{\mathrm{e}}\right)\right)-\frac{5}{2} \mathrm{i}\left\langle\frac{\tilde{\mathrm{B}}_{\|}}{\mathrm{B}}\right\rangle\left[\omega-\omega_{*_{\mathrm{e}}}\left(1+2 \eta_{\mathrm{e}}\right)\right] \\
& \quad-\chi_{\perp \mathrm{e}}^{\text {neo }}\left[\frac{\mathrm{e} \Psi^{\prime \prime}}{\mathrm{T}_{\mathrm{e}}}\left(1-\frac{\omega_{*}}{\omega}\left(1+\frac{\eta_{\mathrm{e}}}{2}\right)\right)-\frac{\hat{\mathrm{n}}^{\prime \prime}}{\mathrm{n}_{\mathrm{e}}}-\frac{\hat{\mathrm{T}}^{\prime \prime}}{2 \mathrm{~T}_{\mathrm{e}}}\right] \\
& =\left(\frac{\mathrm{Inq^{ \prime } \mathrm { x }}}{\mathrm{q}\left\langle\mathrm{B}^{2}\right\rangle^{1 / 2}}\left\langle\frac{1}{\mathrm{R}^{2}}\right\rangle\right)^{2} \frac{\mathrm{f}_{\mathrm{c}}}{\left(1-0.37 \mathrm{f}_{\mathrm{c}}\right)} \frac{\mathrm{T}_{\mathrm{e}}}{\mathrm{m}_{\mathrm{e}} v_{0}}\left(\left(9.61-2.77 \mathrm{f}_{\mathrm{c}}\right) \frac{\hat{\mathrm{n}}_{\mathrm{e}}}{\mathrm{n}_{\mathrm{e}}}+\left(17.15-5.47 \mathrm{f}_{\mathrm{c}}\right) \frac{\hat{\mathrm{T}}_{\mathrm{e}}}{\mathrm{T}_{\mathrm{e}}}\right)
\end{aligned}
$$


where $\chi_{\perp \mathrm{e}}^{\text {neo }} \sim \mathrm{D}_{\perp \mathrm{e}}^{\text {nee }}$ is the neoclassical electron thermal conductivity. Ignoring the small neoclassical electron cross-field transport allows us to solve these equations algebraically to obtain explicit expressions for $\hat{\mathrm{n}}_{\mathrm{e}}$ and $\hat{\mathrm{T}}_{\mathrm{e}}$ :

$$
\begin{aligned}
& -\mathrm{D} \frac{\hat{\mathrm{n}}_{\mathrm{e}}}{\mathrm{n}_{\mathrm{e}}}=\left(1+\lambda_{4} \mathrm{~s}^{2}-\lambda_{2} \mathrm{~s}^{2}\right) \frac{\mathrm{e}}{\mathrm{T}_{\mathrm{e}}}\langle\Phi-\Psi\rangle\left(1-\frac{\omega_{*_{\mathrm{e}}}}{\omega}\right)+\lambda_{2} \mathrm{~s}^{2} \frac{\mathrm{e}}{\mathrm{T}_{\mathrm{e}}}\langle\Phi-\Psi\rangle \frac{\omega_{*_{\mathrm{e}}}}{\omega} \eta_{\mathrm{e}} \\
& -\left\langle\frac{\tilde{\mathrm{B}}_{\|}}{\mathrm{B}}\right\rangle\left(1-\frac{\omega_{*_{\mathrm{e}}}}{\omega}\right)\left[1+\lambda_{4} \mathrm{~s}^{2}-\frac{5}{3} \lambda_{2} \mathrm{~s}^{2}\right]+\left\langle\frac{\tilde{\mathrm{B}}_{\|}}{\mathrm{B}}\right\rangle \frac{\omega_{*_{\mathrm{e}}}}{\omega} \eta_{\mathrm{e}}\left[1+\lambda_{4} \mathrm{~s}^{2}-\frac{10}{3} \lambda_{2} \mathrm{~s}^{2}\right]
\end{aligned}
$$

and

$$
\begin{aligned}
& -\mathrm{D} \frac{\hat{\mathrm{T}}_{\mathrm{e}}}{\mathrm{T}_{\mathrm{e}}}=\left(\lambda_{1} \mathrm{~s}^{2}-\lambda_{2} \mathrm{~s}^{2}\right) \frac{\mathrm{e}}{\mathrm{T}_{\mathrm{e}}}\langle\Phi-\Psi\rangle\left(1-\frac{\omega_{*_{\mathrm{e}}}}{\omega}\right)-\left(1+\lambda_{1} \mathrm{~s}^{2}\right) \frac{\mathrm{e}}{\mathrm{T}_{\mathrm{e}}}\langle\Phi-\Psi\rangle \frac{\omega_{*_{\mathrm{e}}}}{\omega} \eta_{\mathrm{e}} \\
& -\left\langle\frac{\tilde{\mathrm{B}}_{\|}}{\mathrm{B}}\right\rangle\left(1-\frac{\omega_{*_{\mathrm{e}}}}{\omega}\right)\left[\frac{2}{3}+\frac{5}{3} \lambda_{1} \mathrm{~s}^{2}-\lambda_{3} \mathrm{~s}^{2}\right]+\left\langle\frac{\tilde{\mathrm{B}}_{\|}}{\mathrm{B}}\right\rangle \eta_{\mathrm{e}} \frac{\omega_{*_{\mathrm{e}}}}{\omega}\left[\frac{7}{3}+\frac{10}{3} \lambda_{1} \mathrm{~s}^{2}-\lambda_{3} \mathrm{~s}^{2}\right]
\end{aligned}
$$

where

$$
\begin{gathered}
\mathrm{D}=\left\{1+\left(18.55-6.05 \mathrm{f}_{\mathrm{c}}\right) \mathrm{s}^{2}+\left(15.90-7.59 \mathrm{f}_{\mathrm{c}}+0.63 \mathrm{f}_{\mathrm{c}}^{2}\right) \mathrm{s}^{4}\right\} \equiv 1+\mathrm{d}_{0} \mathrm{~s}^{2}+\mathrm{d}_{1} \mathrm{~s}^{4}, \\
\lambda_{1}=\left(2.44-0.45 \mathrm{f}_{\mathrm{c}}\right), \quad \lambda_{2}=\left(5.94-1.69 \mathrm{f}_{\mathrm{c}}\right), \\
\lambda_{3}=\left(6.41-1.85 \mathrm{f}_{\mathrm{c}}\right), \quad \lambda_{4}=\left(11.43-3.65 \mathrm{f}_{\mathrm{c}}\right)
\end{gathered}
$$

and the semi-collisional effects are represented through

$$
\mathrm{s}^{2} \equiv \frac{\mathrm{i}\left(\operatorname{Inq} \mathrm{I}^{\prime}\left\langle\frac{1}{\mathrm{R}^{2}}\right\rangle\right)^{2}}{\mathrm{q}^{2}\left\langle\mathrm{~B}^{2}\right\rangle}\left(\frac{\mathrm{T}_{\mathrm{e}}}{\mathrm{m}_{\mathrm{e}} \omega \mathrm{v}_{0}}\right)\left(\frac{\mathrm{f}_{\mathrm{c}}}{1-0.37 \mathrm{f}_{\mathrm{c}}}\right)
$$




\section{(ii) Ion Solution}

We consider the 'collisional' case, $v_{\mathrm{i}}>\omega$ We again find solutions analogous to the electron eqns. (22) and (23) for $\mathrm{h}_{0}$ and $\mathrm{h}_{1}$, where we again drop the suffices $\mathrm{i}$ on $\mathrm{h}_{\mathrm{i}}$. The equation for $\mathrm{h}_{2}$ is

$$
\mathrm{v}_{\|} \hat{\nabla}_{\|} \mathrm{h}_{2}+\mathrm{v}_{\|} \hat{\nabla}_{\|}\left(\frac{\mathrm{Iv}_{\|}}{\Omega_{\mathrm{i}}}\right) \frac{\partial \mathrm{h}_{1}}{\partial \psi}=\mathrm{C}_{\mathrm{i}}\left(\mathrm{h}_{1}\right)
$$

which provides the constraint to determine $\overline{\mathrm{h}}_{1}$

$$
\left\langle\frac{\mathrm{B}}{\mathrm{v}_{\|}} \mathrm{C}_{\mathrm{i}}\left(\mathrm{h}_{1}\right)\right\rangle=0
$$

For the ions we also take a model pitch-angle scattering collision operator that conserves momentum [15], as in eqn. (26):

$$
\mathrm{C}_{\mathrm{i}}\left(\mathrm{h}_{1}\right)=\frac{2 \mathrm{v}_{\mathrm{ii}}(\mathrm{v}) \mathrm{v}_{\|}}{\mathrm{Bv}} \frac{\partial}{\partial \lambda} \lambda \mathrm{v}_{\|} \frac{\partial}{\partial \lambda} \mathrm{h}_{1}+\mathrm{v}_{\mathrm{ii}}(\mathrm{v}) \mathrm{v}_{\|} \mathrm{f}_{0 \mathrm{i}} \frac{\mathrm{m}_{\mathrm{i}}}{\mathrm{T}_{\mathrm{i}}} \mathrm{u}_{\| \mathrm{i}}^{*}
$$

where

$$
\begin{gathered}
u_{\| i}^{*}=\frac{T_{i}}{m_{i}} \frac{\int d^{3} v v_{i i}(v) v_{\|} h_{1}}{\int d^{3} v v_{i i}(v) v_{\|}^{2} f_{0 i}} \\
v_{i i}(v)=v_{i} \phi(u) / u^{3} ; \quad v_{i}=\frac{\sqrt{2} \pi n_{e} e^{4} \ln \Lambda}{m_{i}^{1 / 2} T_{i}^{3 / 2}}: u=m_{i} v^{2} / 2 T_{i}
\end{gathered}
$$

and the functional form of $\phi(u)$ can be found in eqn. (28). The constraint (43) yields

$$
\mathrm{h}_{1}=\frac{\mathrm{I}}{\Omega_{\mathrm{i}}}\left(\mathrm{v}_{\|}-\frac{\mathrm{B}}{\left\langle\mathrm{B}^{2}\right\rangle^{1 / 2}} \hat{\mathrm{V}}_{\|}\right)\left[\frac{\mathrm{e}}{\mathrm{T}_{\mathrm{i}}}\left(1-\frac{\omega_{*_{\mathrm{i}}}^{\mathrm{T}}}{\omega}\right) \Psi^{\prime} \mathrm{f}_{0 \mathrm{i}}-\mathrm{h}_{0}^{\prime}\right]+\frac{\left\langle\mathrm{u}_{\| \mathrm{i}}^{*} \mathrm{~B}\right\rangle}{\left\langle\mathrm{B}^{2}\right\rangle} \mathrm{f}_{0 \mathrm{i}} \frac{\mathrm{m}_{\mathrm{i}}}{\mathrm{T}_{\mathrm{i}}} \hat{\mathrm{V}}_{\|}
$$

Solving self-consistently for $\left\langle\mathrm{u}_{\| \mathrm{i}}^{*} \mathrm{~B}\right\rangle$ we obtain, on using the result $\gamma=\int_{0}^{\infty} v_{\mathrm{i}} \mathrm{t}^{5 / 2} \mathrm{e}^{-\mathrm{t}} \mathrm{dt} / \int_{0}^{\infty} v_{\mathrm{i}} \mathrm{t}^{3 / 2} \mathrm{e}^{-\mathrm{t}} \mathrm{dt} \simeq 1.33$ (so that $\gamma-3 / 2=0.17$ ) arising from integrals over $\mathrm{v}$ of $v_{\mathrm{ii}}(\mathrm{v})$ given in Ref. 15: 


$$
\left\langle\mathrm{u}_{\| \mathrm{i}}^{*} \mathrm{~B}\right\rangle=-\frac{\mathrm{IB}}{\Omega_{\mathrm{i}}} \frac{\mathrm{T}_{\mathrm{i}}}{\mathrm{m}_{\mathrm{i}}}\left\{\frac{\mathrm{e} \Psi^{\prime}}{\mathrm{T}_{\mathrm{i}}}\left[1-\frac{\omega_{*_{\mathrm{i}}}}{\omega}\left(1-0.17 \eta_{\mathrm{i}}\right)\right]+\frac{\hat{\mathrm{n}}_{\mathrm{i}}^{\prime}}{\mathrm{n}_{\mathrm{i}}}-0.17 \frac{\hat{\mathrm{T}}_{\mathrm{i}}^{\prime}}{\mathrm{T}_{\mathrm{i}}}\right\}
$$

Then

$$
\mathrm{u}_{\| \mathrm{i}}=-\frac{\mathrm{I}}{\Omega_{\mathrm{i}}} \frac{\mathrm{T}_{\mathrm{i}}}{\mathrm{m}_{\mathrm{i}}}\left\{\frac{\mathrm{e} \Psi^{\prime}}{\mathrm{T}_{\mathrm{i}}}\left[1-\frac{\omega_{* \mathrm{i}}}{\omega}\left(1+\eta_{\mathrm{i}}\right)\right]+\frac{\hat{\mathrm{n}}_{\mathrm{i}}^{\prime}}{\mathrm{n}_{\mathrm{i}}}+\frac{\hat{\mathrm{T}}_{\mathrm{i}}^{\prime}}{\mathrm{T}_{\mathrm{i}}}\right\}\left(1-\frac{\mathrm{B}^{2}}{\left\langle\mathrm{~B}^{2}\right\rangle} \mathrm{f}_{\mathrm{c}}\right)+\frac{\left\langle\mathrm{u}_{\| \mathrm{i}}^{*} \mathrm{~B}\right\rangle}{\mathrm{B}} \frac{\mathrm{B}^{2}}{\left\langle\mathrm{~B}^{2}\right\rangle} \mathrm{f}_{\mathrm{c}}
$$

so that, using eqn. (47) to eliminate $\left\langle u_{\| i}^{*} B\right\rangle$,

$$
\mathrm{u}_{\| \mathrm{i}}=-\frac{\mathrm{I}}{\Omega_{\mathrm{i}}} \frac{\mathrm{T}_{\mathrm{i}}}{\mathrm{m}_{\mathrm{i}}}\left\{\frac{\mathrm{e} \Psi^{\prime}}{\mathrm{T}_{\mathrm{i}}}\left[1-\frac{\omega_{*_{\mathrm{i}}}}{\omega}\left(1+\eta_{\mathrm{i}}\right)\right]+\frac{\hat{\mathrm{n}}_{\mathrm{i}}{ }^{\prime}}{\mathrm{n}_{\mathrm{i}}}+\frac{\hat{\mathrm{T}}_{\mathrm{i}}^{\prime}}{\mathrm{T}_{\mathrm{i}}}\right\}+1.17 \frac{\mathrm{I}}{\Omega_{\mathrm{i}}} \frac{\mathrm{T}_{\mathrm{i}}}{\mathrm{m}_{\mathrm{i}}} \frac{\mathrm{B}^{2}}{\left\langle\mathrm{~B}^{2}\right\rangle} \mathrm{f}_{\mathrm{c}}\left\{\frac{\hat{\mathrm{T}}_{\mathrm{i}}^{\prime}}{\mathrm{T}_{\mathrm{i}}}-\frac{\omega_{*_{\mathrm{i}}} \eta_{\mathrm{i}}}{\omega} \frac{\mathrm{e} \Psi^{\prime}}{\mathrm{T}_{\mathrm{i}}}\right\}
$$

This result justifies the neglect of the term in $\mathrm{O}\left(\mathrm{k}_{\|} \mathrm{u}_{\| \mathrm{i}} / \omega\right)$ on the right-hand sides of eqns. (35) and (37). Equations (32) and (49) will allow us to calculate $\mathrm{j}_{\|}$later.

The equation for $\mathrm{h}_{3}$ appears in next order:

$$
\begin{aligned}
& \mathrm{v}_{\|} \hat{\nabla}_{\|} \mathrm{h}_{3}+\mathrm{v}_{\|} \hat{\nabla}_{\|}\left(\frac{\mathrm{Iv}_{\|}}{\Omega_{\mathrm{i}}}\right) \frac{\partial \mathrm{h}_{2}}{\partial \psi}+\frac{\mathrm{innq}^{\prime} \mathrm{x}}{\mathrm{R}^{2} \mathrm{~Bq}} \mathrm{v}_{\|} \mathrm{h}_{0}-\mathrm{i} \omega \mathrm{h}_{0} \\
& =\mathrm{C}_{\mathrm{i}}\left(\mathrm{h}_{2}\right)+\frac{\mathrm{ie}}{\mathrm{T}_{\mathrm{i}}} \mathrm{f}_{0 \mathrm{i}}\left(\omega-\omega_{*_{\mathrm{i}}}^{\mathrm{T}}\right)\left(\Phi-\Psi+\frac{\mathrm{v}_{\perp}^{2} \tilde{\mathrm{B}}_{\|}}{2 \Omega_{\mathrm{i}}}\right)
\end{aligned}
$$

where we have taken the $z_{i} \rightarrow 0$ limit of the Bessel functions. We apply the operator (34) which annihilates the first terms on both the left and the right to obtain

$$
\omega \hat{\mathrm{n}}_{\mathrm{i}}=\mathrm{n}_{\mathrm{i}}\left[\frac{\mathrm{e}\langle\Phi-\Psi\rangle}{\mathrm{T}_{\mathrm{i}}}\left(\omega-\omega_{*_{\mathrm{i}}}\right)+\left\langle\frac{\tilde{\mathrm{B}}_{\|}}{\mathrm{B}}\right\rangle\left(\omega-\omega_{*_{\mathrm{i}}}\left(1+\eta_{\mathrm{i}}\right)\right)\right]
$$

Again a similar equation for the ion energy balance can be derived. More significantly, however, there is now a contribution from $\mathrm{C}_{\mathrm{i}}\left(\mathrm{h}_{2}\right)$ : this corresponds to ion neoclassical cross-field transport. Following Ref. 16 one obtains 


$$
\begin{aligned}
\frac{3 \mathrm{i}}{2} \omega\left(\frac{\hat{\mathrm{n}}_{\mathrm{i}}}{\mathrm{n}_{\mathrm{i}}}+\frac{\hat{\mathrm{T}}_{\mathrm{i}}}{\mathrm{T}_{\mathrm{i}}}\right)= & \frac{3 \mathrm{i}}{2} \frac{\mathrm{e}\langle\Phi-\Psi\rangle}{\mathrm{T}_{\mathrm{i}}}\left[\omega-\omega_{*_{\mathrm{i}}}\left(1+\eta_{\mathrm{i}}\right)\right]+\frac{5 \mathrm{i}}{2}\left\langle\frac{\tilde{\mathrm{B}}_{\|}}{\mathrm{B}}\right\rangle\left(\omega-\omega_{*_{\mathrm{i}}}\left(1+2 \eta_{\mathrm{i}}\right)\right) \\
& +\frac{\mathrm{T}_{\mathrm{i}} \mathrm{I}^{2}}{\mathrm{~m}_{\mathrm{i}} \Omega_{\mathrm{i} 0}^{2} \tau_{\mathrm{ii}}}\left\langle\frac{\left\langle\mathrm{B}^{2}\right\rangle}{\mathrm{B}^{2}}-\mathrm{f}_{\mathrm{c}}\right\rangle\left[\frac{\omega_{*_{\mathrm{i}}}}{\omega} \eta_{\mathrm{i}} \frac{\mathrm{e} \Phi^{\prime \prime}}{\mathrm{T}_{\mathrm{i}}}-\frac{\hat{\mathrm{T}}_{\mathrm{i}}^{\prime \prime}}{\mathrm{T}_{\mathrm{i}}}\right]
\end{aligned}
$$

Equations (51) and (52) provide an explicit expression for $\hat{\mathrm{T}}_{\mathrm{i}}$ :

$$
\begin{aligned}
\hat{\mathrm{T}}_{\mathrm{i}}-\frac{2 \mathrm{i}}{3} & \frac{\mathrm{T}_{\mathrm{i}} \mathrm{I}^{2}}{\mathrm{~m}_{\mathrm{i}} \Omega_{\mathrm{i} 0}^{2} \tau_{\mathrm{ii}} \omega}\left\langle\frac{\left\langle\mathrm{B}^{2}\right\rangle}{\mathrm{B}^{2}}-\mathrm{f}_{\mathrm{c}}\right\rangle \hat{\mathrm{T}}_{\mathrm{i}}^{\prime \prime}=-\mathrm{e}\langle\Phi-\Psi\rangle \frac{\eta_{\mathrm{i}} \omega_{*_{\mathrm{i}}}}{\omega} \\
& -\frac{2 \mathrm{i}}{3} \frac{\mathrm{T}_{\mathrm{i}} \mathrm{I}^{2}}{\mathrm{~m}_{\mathrm{i}} \Omega_{\mathrm{i} 0}^{2} \tau_{\mathrm{ii}} \omega}\left\langle\frac{\left\langle\mathrm{B}^{2}\right\rangle}{\mathrm{B}^{2}}-\mathrm{f}_{\mathrm{c}}\right\rangle \frac{\omega_{*_{\mathrm{i}}}}{\omega} \eta_{\mathrm{i}} \mathrm{e} \Phi^{\prime \prime}+\frac{2}{3} \mathrm{~T}_{\mathrm{i}}\left\langle\frac{\tilde{\mathrm{B}}_{\|}}{\mathrm{B}}\right\rangle\left[\left(1-\frac{\omega_{*_{\mathrm{i}}}}{\omega}\right)-\frac{7}{2} \frac{\eta_{\mathrm{i}} \omega_{*_{\mathrm{i}}}}{\omega}\right]
\end{aligned}
$$

The equations for the ion quantities $\hat{\mathrm{n}}_{\mathrm{i}}$ and $\hat{\mathrm{T}}_{\mathrm{i}}$ are linearly algebraic if we ignore ion neoclassical transport, otherwise they involve a second order ordinary differential equation (ODE).

\section{Maxwell's Equations}

To obtain equations for the perturbed fields we utilise quasi-neutrality and the parallel and perpendicular Ampère's equations for $\Psi$ and $\tilde{B}_{\|}$, using the perturbed charges and currents calculated from the gyro-kinetic solutions.

First, quasi-neutrality implies

$$
\tilde{\mathrm{n}}_{\mathrm{e}}=\tilde{\mathrm{n}}_{\mathrm{i}}
$$

where

$$
\tilde{n}_{j}=-\frac{n_{j} e_{j}}{T_{j}} \Phi+\frac{n_{j} e_{j}}{T_{j}}\left(1-\frac{\omega_{* j}}{\omega}\right) \Psi+\hat{n}_{j}
$$

Thus eqn. (54) relates $\Phi$ to $\Psi$ and $\hat{\mathrm{n}}_{\mathrm{i}, \mathrm{e}}$; the $\hat{\mathrm{n}}_{\mathrm{i}, \mathrm{e}}$ are independent of $\theta$ and we will see below when considering Ampère's equations for $\Psi$, that $\Psi$ is also independent of $\theta$ in leading order, implying the same is true for $\Phi$. 
Ampère's laws for $\mathrm{A}_{\|}$and $\tilde{\mathrm{B}}_{\|}$provide relationships to eliminate $\tilde{\mathrm{B}}_{\|}$and $\Psi$ from our equations. To evaluate $\tilde{\mathrm{B}}_{\|}$we must calculate the perpendicular current arising from the first order in a Larmor radius expansion of the gyro-phase factor $\exp \left(i \mathrm{i}_{\mathrm{i}}\right)$ in eqn. (2) and use it in Ampère's law. We find $[5,14]$

$$
\frac{\tilde{\mathrm{B}}_{\|}}{\mathrm{B}}=-\frac{\mu_{0} \tilde{\mathrm{p}}}{\mathrm{B}^{2}}
$$

where we have used the definitions (10) and (21) of $g_{j}$ and $h_{j}$ which imply

$$
\tilde{\mathrm{p}}=\sum_{\mathrm{j}} \tilde{\mathrm{p}}_{\mathrm{j}}=\hat{\mathrm{p}}+\frac{\mathrm{n}}{\omega} \mathrm{p}_{0}^{\prime} \Psi ; \quad \hat{\mathrm{p}}=\sum_{\mathrm{j}} \hat{\mathrm{p}}_{\mathrm{j}}
$$

$\tilde{\mathrm{B}}_{\|}$can normally be neglected but will be seen to play a significant role in converting $\nabla B$ drifts to curvature drift in the vorticity equation discussed in the next section.

In the case of $\mathrm{A}_{\|}$we have

$$
-|\nabla \psi|^{2} \frac{\partial^{2}}{\partial \mathrm{x}^{2}} \mathrm{~A}_{\|}=\mu_{0} \mathrm{j}_{\|}
$$

where we must use the neoclassical current for $j_{\|}$. In lowest order, expressing $A_{\|}$in terms of $\Psi$ and writing $\Psi=\Psi^{(0)}+\Psi^{(1)}+\ldots$ where the expansion parameter is $\eta=\left(1 / \beta_{\mathrm{e}}\right)\left(\mathrm{L}_{\mathrm{s}} / \mathrm{L}_{\mathrm{n}}\right)^{2}\left(v_{\mathrm{e}} / \Omega_{\mathrm{e}}\right)^{2}$, we have

$$
\frac{|\nabla \psi|^{2}}{\mathrm{i} \omega} \frac{\mathrm{I}}{\mathrm{R}^{2} \mathrm{~Bq}} \frac{\partial^{2}}{\partial \mathrm{x}^{2}} \frac{\partial}{\partial \theta} \Psi^{(0)}=0 \quad \Rightarrow \quad \Psi^{(0)}=\Psi^{(0)}(\mathrm{x})
$$

while in second order

$$
-\frac{|\nabla \psi|^{2}}{i \omega} \frac{\mathrm{I}}{\mathrm{R}^{2} \mathrm{~Bq}} \frac{\partial^{2}}{\partial \mathrm{x}^{2}}\left(\frac{\partial \Psi^{(1)}}{\partial \theta}+\mathrm{inq}^{\prime} \mathrm{x}^{(0)}(\mathrm{x})\right)=\mu_{0} \mathrm{j}_{\|}
$$

This imposes a solubility condition

$$
\frac{\mathrm{nq}^{\prime} \mathrm{I}}{\omega}\left\langle\frac{1}{\mathrm{R}^{2}}\right\rangle \frac{\mathrm{d}^{2}\left(\mathrm{x} \Psi^{(0)}\right)}{\mathrm{dx}^{2}}=-\mu_{0}\left\langle\frac{\mathrm{j}_{\|} \mathrm{B}}{|\nabla \psi|^{2}}\right\rangle
$$


Using eqns. (32) and (49) to evalulate the neoclassical parallel current we can calculate the above average of $j_{\|}$. Writing $j_{\|}$as a sum of an inductive contribution driven by $E_{\|}$ (see eqn. (12)) and the 'bootstrap' current driven by radial plasma gradients, we have

$$
\mathrm{j}_{\|}=\mathrm{j}_{\|}^{\text {inductive }}+\mathrm{j}_{\|}^{\text {bootstrap }}
$$

with

$$
\begin{aligned}
& \left\langle\frac{\mathrm{j}_{\|}^{\text {inductive }} \mathrm{B}}{|\nabla \psi|^{2}}\right\rangle \\
& =\frac{\text { inq'xI }^{\prime}}{\mathrm{q}} \frac{\left\langle 1 / \mathrm{R}^{2}\right\rangle\left\langle\mathrm{B}^{2} /|\nabla \psi|^{2}\right\rangle}{\left\langle\mathrm{B}^{2}\right\rangle}\left(\frac{\mathrm{n}_{\mathrm{e}} \mathrm{e}^{2}}{\mathrm{~m}_{\mathrm{e}} \mathrm{v}_{0}}\right) \frac{\mathrm{f}_{\mathrm{c}}}{\left(1-0.37 \mathrm{f}_{\mathrm{c}}\right)} \frac{\mathrm{T}_{\mathrm{e}}}{\mathrm{e}}\left[\left(2.45-0.45 \mathrm{f}_{\mathrm{c}}\right) \frac{\hat{\mathrm{n}}_{\mathrm{e}}}{\mathrm{n}_{\mathrm{e}}}+\left(5.94-1.69 \mathrm{f}_{\mathrm{c}}\right) \frac{\hat{\mathrm{T}}_{\mathrm{e}}}{\mathrm{T}_{\mathrm{e}}}\right]
\end{aligned}
$$

$$
\left\langle\frac{\mathrm{j}_{\|}^{\text {bootstrap }} \mathrm{B}}{|\nabla \psi|^{2}}\right\rangle=\mathrm{Ip}^{\prime}\left[\left\langle\frac{1}{|\nabla \psi|^{2}}\right\rangle-\frac{\left\langle\mathrm{B}^{2} /|\nabla \psi|^{2}\right\rangle}{\left\langle\mathrm{B}^{2}\right\rangle}\right]+\mathrm{I} \frac{\left\langle\mathrm{B}^{2} /|\nabla \psi|^{2}\right\rangle}{\left\langle\mathrm{B}^{2}\right\rangle} \frac{\left(1-\mathrm{f}_{\mathrm{c}}\right)}{\left(1-0.37 \mathrm{f}_{\mathrm{c}}\right)} \tilde{\mathrm{J}}
$$

where

$$
\tilde{\mathrm{J}}=-\alpha_{\mathrm{n}} \tilde{\mathrm{n}}_{\mathrm{e}}^{\prime}\left(\mathrm{T}_{\mathrm{e}}+\mathrm{T}_{\mathrm{i}}\right)-\alpha_{\mathrm{e}} \mathrm{n}_{\mathrm{e}} \tilde{\mathrm{T}}_{\mathrm{e}}^{\prime}+\alpha_{\mathrm{i}} \mathrm{n}_{\mathrm{i}} \tilde{\mathrm{T}}_{\mathrm{i}}^{\prime}
$$

with

$$
\alpha_{n}=1.06-0.06\left(1-f_{c}\right), \alpha_{e}=0.55+0.45\left(1-f_{c}\right), \alpha_{i}=0.183-1.25\left(1-f_{c}\right)+0.066\left(1-f_{c}\right)^{2}
$$

where we have introduced the total perturbations $\tilde{n}_{e, i}$ and $\tilde{T}_{e, i}$ in calculating the $j_{\|}^{\text {bootstrap }}$ contribution, but retained $\hat{\mathrm{n}}_{\mathrm{e}}$ and $\hat{\mathrm{T}}_{\mathrm{e}}$ in the inductive term since these are manifestly proportional to $E_{\|}$, as can be seen from eqns. (12), (38) and (39) when $\tilde{B}_{\|}$is ignored. (Note we have included the Pfirsch-Schlüter contribution in eqn. (64) so this is strictly the pressure gradient driven current, rather than just the bootstrap current.) Here the $\tilde{\mathrm{n}}_{\mathrm{j}}$ are defined in eqn. (55) and

$$
\tilde{\mathrm{T}}_{\mathrm{j}}=\hat{\mathrm{T}}_{\mathrm{j}}+\frac{\mathrm{n}}{\omega} \mathrm{T}_{0 \mathrm{j}}{ }^{\prime} \Psi
$$


It is convenient to write the total $\mathrm{j}_{\|}$in the form

$$
\begin{aligned}
\left\langle\frac{\mathrm{j}_{\|} \mathrm{B}}{|\nabla \psi|^{2}}\right\rangle= & -\frac{\operatorname{inI} \sigma_{\|}^{\mathrm{sc}}\left(\mathrm{s}^{2}\right)}{\mu_{0} \mathrm{p}_{0}^{\prime}}\left(\frac{\mathrm{q}^{\prime}}{\mathrm{q}}\right)^{2}\left\langle\frac{1}{\mathrm{R}^{2}}\right\rangle^{2} \mathrm{Lx}\left(\Phi^{(0)}-\Psi^{(0)}\right)+\frac{\mathrm{Iq}^{\prime}}{\mathrm{q} \mu_{0} \mathrm{p}_{0}^{\prime}}\left\langle\frac{1}{\mathrm{R}^{2}}\right\rangle \mathrm{H}^{\prime} \\
& -\frac{\left(1-\mathrm{f}_{\mathrm{c}}\right)}{\left(1-0.37 \mathrm{f}_{\mathrm{c}}\right)}\left(\frac{\mathrm{Iq}}{\mathrm{q} \mu_{0} \mathrm{p}_{0}^{\prime}}\right)\left\langle\frac{1}{\mathrm{R}^{2}}\right\rangle \mathrm{L}\left[\alpha_{\mathrm{n}} \tilde{\mathrm{n}}_{\mathrm{e}}^{\prime}\left(\mathrm{T}_{\mathrm{e}}+\mathrm{T}_{\mathrm{i}}\right)+\alpha_{\mathrm{e}} \mathrm{n} \tilde{\mathrm{T}}_{\mathrm{e}}^{\prime}-\alpha_{\mathrm{i}} \mathrm{n} \tilde{\mathrm{T}}_{\mathrm{i}}^{\prime}\right]
\end{aligned}
$$

where $\mathrm{H}$ is a flux-surface-averaged equilibrium quantity introduced in Ref. 13 and $\mathrm{L}=\left(\mu_{0} \mathrm{p}_{0}^{\prime} \mathrm{q} / \mathrm{q}^{\prime}\left\langle 1 / \mathrm{R}^{2}\right\rangle\left\langle\mathrm{B}^{2}\right\rangle\right)\left\langle\mathrm{B}^{2} /|\nabla \psi|^{2}\right\rangle ; \mathrm{L}$ was first introduced in Refs. 2 and 5 (these quantities are also defined in Appendix A). The neoclassical semi-collisional conductivity $\sigma_{\|}^{\text {sc }}$ is given by:

where

$$
\sigma_{\|}^{\mathrm{sc}}=\frac{\sigma_{0}}{\mathrm{D}} \frac{\mathrm{f}_{\mathrm{c}}}{1-0.37 \mathrm{f}_{\mathrm{c}}}\left\{\left(1-\omega_{*_{\mathrm{e}}} / \omega\right)\left(\sigma_{\mathrm{n}}+\mathrm{d}_{1} \mathrm{~s}^{2}\right)-\left(\omega *_{\mathrm{e}} \eta_{\mathrm{e}} \sigma_{\mathrm{T}} / \omega\right)\right\}
$$

$$
\sigma_{\mathrm{n}}=2.45-0.45 \mathrm{f}_{\mathrm{c}}, \sigma_{\mathrm{T}}=5.94-1.69 \mathrm{f}_{\mathrm{c}}
$$

with $\sigma_{0}=\left(\mathrm{n}_{\mathrm{e}} \mathrm{e}^{2} / \mathrm{m}_{\mathrm{e}} v_{0}\right)$. These three terms in eqn. (66) represent the parallel current driven by the electric field $\mathrm{E}_{\|}$(with neoclassical semi-collisional conductivity), PfirschSchlüter and bootstrap current contributions, respectively. This expression still fails to reproduce the Spitzer resistivity nor, in the limit of a small fraction of trapped particles, the correct coefficient for the electron temperature gradient contribution to the bootstrap current as discussed in Ref. 16; however it provides a plausible prescription for investigating the bootstrap current at finite aspect ratio. (A more careful treatment using the Spitzer-Härm solution could be invoked [17]; we do not pursue this improvement further in this paper).

Combining eqns. (61) and (66) we obtain a neoclassical Ohm's law:

$$
\begin{aligned}
& \frac{\mathrm{n}}{\omega} \frac{\mathrm{d}^{2}\left(\mathrm{x} \Psi^{(0)}\right)}{\mathrm{dx}^{2}}=-\frac{\text { inq }^{\prime} \sigma_{\|}^{\mathrm{sc}}\left(\mathrm{s}^{2}\right)}{\mathrm{p}_{0}^{\prime} \mathrm{q}}\left\langle\frac{1}{\mathrm{R}^{2}}\right) \operatorname{Lx}\left(\Phi^{(0)}-\Psi^{(0)}\right)+\frac{\mathrm{H}^{\prime}}{\mathrm{p}_{0}^{\prime}} \\
& -\frac{\left(1-\mathrm{f}_{\mathrm{c}}\right)}{\left(1-0.37 \mathrm{f}_{\mathrm{c}}\right)} \frac{\mathrm{L}}{\mathrm{p}_{0}^{\prime}}\left[\alpha_{\mathrm{n}} \tilde{\mathrm{n}}_{\mathrm{e}}^{\prime}\left(\mathrm{T}_{\mathrm{e}}+\mathrm{T}_{\mathrm{i}}\right)+\alpha_{\mathrm{e}} \mathrm{n} \tilde{\mathrm{T}}_{\mathrm{e}}^{\prime}-\alpha_{\mathrm{i}} \mathrm{n} \tilde{\mathrm{T}}_{\mathrm{i}}^{\prime}\right]
\end{aligned}
$$

Equation (68) with eqns. (38) and (39) for $\hat{\mathrm{n}}_{\mathrm{e}}$ and $\hat{\mathrm{T}}_{\mathrm{e}}$ and eqns. (51) and (53) for $\hat{\mathrm{n}}_{\mathrm{i}}$ and $\hat{\mathrm{T}}_{\mathrm{i}}$ provides a relationship between $\Psi^{(0)}$ and $\Phi$ that includes the effects of neoclassical resistivity and the bootstrap current: 


\section{The Vorticity Equation}

To close the system of equations we use the vorticity equation in the long wavelength limit of the ion finite Larmor radius in the Bessel functions [5]. In lowest order we will confirm $\Psi^{(0)}$ is flute-like and in first order we obtain an equation for $\Psi^{(1)}$; this can be solved, with constants of integration being determined by poloidal periodicity constraints. Finally in second order, the solubility condition on $\Psi^{(2)}$ provides a fluxsurface-averaged equation for $\Psi^{(0)}$ after substituting for $\Psi^{(1)}$. However this also involves flux-surface averages of velocity moments of the magnetic drift term in the gyro-kinetic equations which can be evaluated by repeated integrations by parts in poloidal angle, use of the gyro-kinetic equations up to third order and noting conservation of momentum in ion-ion collisions. These manipulations give rise to terms that can be recognised as: (i) the enhanced neoclassical ion inertia which adds to that already present in the vorticity equation due to the usual ion polarisation drift; (ii) ion neoclassical crossfield viscosity, dominated by the perturbed ion temperature gradient; and (iii) a term arising from the parallel gradient of perturbed pressure. This equation will provide another second order ODE linking $\Phi^{(0)}$ and $\Psi^{(0)}$ that also involves $\hat{\mathrm{p}}$ and $\hat{\mathrm{p}}^{\prime}$.

To obtain the vorticity equation we operate on the gyro-kinetic equations (1) with

$\sum_{j} e_{j} \int d^{3} v$ to obtain:

$\frac{i}{\omega} \mathbf{B} \cdot \nabla\left(\frac{\mathrm{j}_{\|}}{B}\right)=-\sum_{\mathrm{j}} \frac{\mathrm{e}_{\mathrm{j}}^{2}}{\mathrm{~T}_{\mathrm{j}}} \mathrm{d}^{3} \mathrm{vf}_{0 \mathrm{j}}\left\{\left[1-\left(1-\frac{\omega_{\mathrm{k}_{\mathrm{j}}}^{\mathrm{T}}}{\omega}\right) \mathrm{J}_{0}^{2}\right] \Phi-\left(1-\frac{\omega_{\mathrm{k}_{\mathrm{j}}}^{\mathrm{T}}}{\omega}\right) \mathrm{J}_{0} \mathrm{~J}_{1} \frac{\mathrm{v}_{\perp}}{\mathrm{k}_{\perp}} \frac{\tilde{\mathrm{B}}_{\|}}{\mathrm{B}}\right\}$

$-i \sum_{j} e_{j} \int d^{3} v_{0} \frac{\mathbf{v}_{d j} \nabla g_{j}}{\omega}+\frac{i}{\omega} \sum_{j} e_{j} \int d^{3} v \sigma\left|v_{\|}\right| b . \nabla J_{0} g_{j}$

In the long wavelength limit, $z_{j} \ll 1$, and using Maxwell's equation (58) in terms of $\Psi$, this can be written as

$$
\begin{aligned}
& \frac{B}{\omega^{2}} \nabla_{\|} \frac{|\nabla \psi|^{2}}{\mu_{0} B} \frac{\partial^{2}}{\partial x^{2}} \nabla_{\|} \Psi=\sum_{j} \frac{e_{j}^{2}}{T_{j}} \int d^{3} v f_{o j}\left\{\frac{\omega_{* j}^{T}}{\omega} \Phi-\left(1-\frac{\omega_{* j}^{T}}{\omega}\right)\left[\frac{m_{j}^{2} v_{\perp}^{2}}{2 e_{j}^{2} B^{2}}|\nabla \psi|^{2} \frac{d^{2} \Phi}{d x^{2}}+\frac{m_{j} v_{\perp}^{2}}{2 e_{j} B} \tilde{B}_{\|}\right]\right\} \\
& +i \sum_{j} e_{j} \int d^{3} v J_{0} \frac{\mathbf{v}_{d j} \cdot \nabla g_{j}}{\omega}-\frac{i}{\omega} \sum_{j} \frac{m_{j}}{e_{j}} \int d^{3} v \sigma\left|v_{\|}\right|_{j} \mathbf{b} \cdot \nabla\left(\frac{m_{j} v_{\perp}^{2}|\nabla \psi|^{2}}{2 B^{2}}\right) g^{\prime \prime}
\end{aligned}
$$

This reduces to 


$$
\begin{gathered}
\frac{\mathrm{I}^{2}}{\omega^{2} \mathrm{R}^{2} \mathrm{q}^{2}}\left(\frac{\partial}{\partial \theta}+i n q^{\prime} \mathrm{x}\right) \frac{|\nabla \psi|^{2}}{\mu_{0} \mathrm{R}^{2} \mathrm{~B}^{2}} \frac{\partial^{2}}{\partial \mathrm{x}^{2}}\left(\frac{\partial}{\partial \theta}+i n q^{\prime} \mathrm{x}\right) \Psi=-\frac{\mathrm{m}_{\mathrm{i}} \mathrm{n}_{\mathrm{i}}}{\mathrm{B}^{2}}\left[1-\frac{\omega_{* \mathrm{i}}}{\omega}\left(1+\eta_{\mathrm{i}}\right)\right]|\nabla \psi|^{2} \frac{\mathrm{d}^{2} \Phi}{\mathrm{dx}^{2}} \\
-\frac{\mathrm{np}_{0}^{\prime}}{\omega} \frac{\tilde{B}_{\|}}{\mathrm{B}}+\frac{\mathrm{i}}{\omega} \sum_{\mathrm{j}} \mathrm{e}_{\mathrm{j}} \int \mathrm{d}^{3} \mathrm{v} \frac{\mathrm{Iv}_{\|}}{\mathrm{R}^{2} \mathrm{~Bq}} \frac{\partial}{\partial \theta}\left(\frac{\mathrm{I} \mathrm{v}_{\|}}{\Omega_{\mathrm{j}}}\right)\left[\frac{\mathrm{e}_{\mathrm{j}}}{\mathrm{T}_{\mathrm{j}}}\left(1-\frac{\omega_{* \mathrm{j}}^{\mathrm{T}}}{\omega}\right) \Psi^{\prime} \mathrm{f}_{0 \mathrm{j}}+\mathrm{h}_{\mathrm{j}}^{\prime}\right] \\
-\sum_{\mathrm{j}} \mathrm{e}_{\mathrm{j}} \int \mathrm{d}^{3} \mathrm{v}\left[\frac{\mathrm{e}_{\mathrm{j}}}{\mathrm{T}_{\mathrm{j}}}\left(1-\frac{\omega_{* \mathrm{j}}^{\mathrm{T}}}{\omega}\right) \Psi \mathrm{f}_{0 \mathrm{j}}+\mathrm{h}_{\mathrm{j}}\right] \frac{\omega_{\mathrm{djn}}}{\omega}-\frac{\mathrm{i}}{\omega} \sum_{\mathrm{j}} \frac{\mathrm{m}_{\mathrm{j}}}{\mathrm{e}_{\mathrm{j}}} \int \mathrm{d}^{3} \mathrm{v} \sigma\left|\mathrm{v}_{\|}\right|_{\mathrm{j}} \mathbf{b} \cdot \nabla\left(\frac{\mathrm{m}_{\mathrm{j}} \mathrm{v}_{\perp}^{2}|\nabla \psi|^{2}}{2 \mathrm{~B}^{2}}\right) \mathrm{g}^{\prime \prime}
\end{gathered}
$$

where the normal curvature term is given by

$$
\begin{aligned}
& \omega_{\mathrm{djn}}=\frac{\mathbf{b}}{\Omega_{\mathrm{j}}} \times\left(\frac{\mathrm{v}_{\perp}^{2}}{2} \nabla \ln \mathrm{B}+\mathrm{v}_{\|}^{2} \mathbf{\kappa}\right) \cdot \nabla(\mathrm{n} \varphi-\mathrm{m} \theta) \\
& =-\frac{\mathrm{nB}}{\Omega_{\mathrm{j}}}\left[\frac{|\nabla \psi|^{2}}{\mathrm{R}^{2} \mathrm{~B}^{2}}\left\{\frac{\mathrm{v}_{\perp}^{2}}{2} \frac{\partial}{\partial \psi} \ln \mathrm{B}+\frac{\mathrm{v}_{\|}^{2}}{\mathrm{~B}^{2}} \frac{\partial}{\partial \psi}\left(\mu_{0} \mathrm{p}+\frac{\mathrm{B}^{2}}{2}\right)\right\}+\left(\frac{\mathrm{v}_{\perp}^{2}}{2}+\mathrm{v}_{\|}^{2}\right) \frac{\nabla \psi \cdot \nabla \theta}{\mathrm{R}^{2} \mathrm{~B}^{2}} \frac{\partial}{\partial \theta} \ln \mathrm{B}\right]
\end{aligned}
$$

We solve this order by order: in lowest order we confirm the result

$$
\frac{\partial^{2}}{\partial \mathrm{x}^{2}} \frac{\partial \Psi^{(0)}}{\partial \theta}=0 \Rightarrow \Psi^{(0)}=\Psi^{(0)}(\mathrm{x})
$$

In first order, inserting $\mathrm{h}_{0 \mathrm{j}}{ }^{\prime}$ on the right-hand side

$$
\begin{gathered}
\frac{\mathrm{I}^{2}}{\omega^{2} \mathrm{R}^{2} \mathrm{q}^{2}} \frac{\partial}{\partial \theta}\left[\frac{|\nabla \psi|^{2}}{\mu_{0} \mathrm{R}^{2} \mathrm{~B}^{2}} \frac{\partial^{2}}{\partial \mathrm{x}^{2}}\left\{\frac{\partial}{\partial \theta} \Psi^{(1)}+i n q^{\prime} \mathrm{x} \Psi^{(0)}\right\}\right] \\
=i \frac{\mathrm{I}^{2}}{\omega \mathrm{R}^{2} \mathrm{q}} \frac{\partial}{\partial \theta}\left(\frac{\tilde{\mathrm{p}}^{\prime}}{\mathrm{B}^{2}}\right)
\end{gathered}
$$

where we have used eqn. (57) so that a first integration yields

$$
\frac{|\nabla \psi|^{2}}{\omega q \mu_{0} \mathrm{R}^{2} \mathrm{~B}^{2}} \frac{\partial^{2}}{\partial \mathrm{x}^{2}}\left[\frac{\partial}{\partial \theta} \Psi^{(1)}+\mathrm{inq}^{\prime} \mathrm{x}^{(0)}\right]=\mathrm{i} \frac{\tilde{\mathrm{p}}^{\prime}}{\mathrm{B}^{2}}+\mathrm{F}(\mathrm{x})
$$

where $\mathrm{F}$ is an arbitrary function. Annihilating $\Psi^{(1)}$ on the left-hand side of eqn. (75), we obtain an equation for $\mathrm{F}(\mathrm{x})$ so that 


$$
\begin{aligned}
& \frac{|\nabla \psi|^{2}}{\omega^{2} \mathrm{q} \mu_{0} \mathrm{R}^{2} \mathrm{~B}^{2}} \frac{\partial^{2}}{\partial \mathrm{x}^{2}}\left[\frac{\partial}{\partial \theta} \Psi^{(1)}+\text { inq }^{\prime} \mathrm{x}^{(0)}\right] \\
& =\frac{\mathrm{i}}{\omega}\left(\frac{1}{\mathrm{~B}^{2}}-\left\langle\frac{1}{|\nabla \psi|^{2}}\right\rangle\left\langle\frac{\mathrm{B}^{2}}{|\nabla \psi|^{2}}\right\rangle^{-1}\right) \tilde{\mathrm{p}}^{\prime}+\frac{\text { inq }^{\prime}}{\mu_{0} \omega^{2} \mathrm{q}}\left\langle\frac{1}{\mathrm{R}^{2}}\right\rangle\left\langle\frac{\mathrm{B}^{2}}{|\nabla \psi|^{2}}\right\rangle^{-1} \frac{\mathrm{d}^{2}}{\mathrm{dx} \mathrm{x}^{2}} \mathrm{x} \Psi^{(0)}
\end{aligned}
$$

Finally, in second order, we have

$$
\begin{aligned}
& \frac{I^{2}}{\omega^{2} R^{2} q^{2}} \frac{\partial}{\partial \theta}\left[\frac{|\nabla \psi|^{2}}{\mu_{0} R^{2} B^{2}} \frac{\partial^{2}}{\partial x^{2}}\left(\frac{\partial}{\partial \theta} \Psi^{(2)}+i n q^{\prime} x^{(1)}\right)\right]+\frac{i n q^{\prime} I^{2}}{\omega^{2} R^{2} q^{2}} \frac{|\nabla \psi|^{2}}{\mu_{0} R^{2} B^{2}} \frac{\partial^{2}}{\partial x^{2}}\left[\frac{\partial}{\partial \theta} \Psi^{(1)}+i n q^{\prime} x \Psi^{(0)}\right] \\
& =-m_{i} n_{i} \frac{|\nabla \psi|^{2}}{B^{2}}\left[1-\frac{\omega_{x_{i}}}{\omega}\left(1+\eta_{\mathrm{i}}\right)\right] \frac{\mathrm{d}^{2} \Phi}{\mathrm{dx}^{2}}-\frac{\mathrm{np}_{0}^{\prime}}{\omega} \frac{\tilde{\mathrm{B}}_{\|}}{\mathrm{B}} \\
& +\frac{\mathrm{n}}{\omega} \frac{\mathrm{p}_{0}^{\prime}}{\omega} \Psi^{(0)}\left[\frac{|\nabla \psi|^{2}}{\mathrm{R}^{2} \mathrm{~B}^{2}} \frac{\partial}{\partial \psi}\left(\mu_{0} \mathrm{p}+\mathrm{B}^{2}\right)+\frac{2 \nabla \psi \cdot \nabla \theta}{\mathrm{R}^{2} \mathrm{~B}^{2}} \frac{\partial}{\partial \theta} \ln \mathrm{B}\right] \\
& +\frac{\mathrm{n}}{\omega} \sum_{\mathrm{j}} \int \mathrm{d}^{3} \mathrm{~h}_{\mathrm{j}}\left\{\frac{\mathrm{m}_{\mathrm{j}} \mathrm{v}_{\perp}^{2}}{2}\left(\frac{2|\nabla \psi|^{2}}{\mathrm{R}^{2} \mathrm{~B}^{2}} \frac{\partial}{\partial \psi}+\frac{\nabla \psi \cdot \nabla \theta}{\mathrm{R}^{2} \mathrm{~B}^{2}} \frac{\partial}{\partial \theta}\right) \ln \mathrm{B}+\mathrm{m}_{\mathrm{j}} \mathrm{v}_{\|}^{2}\left[\frac{2|\nabla \psi|^{2}}{\mathrm{R}^{2} \mathrm{~B}^{2}}\left\{\frac{1}{\mathrm{~B}^{4}} \frac{\partial}{\partial \psi}\left(\mu_{0} \mathrm{p}+\frac{\mathrm{B}^{2}}{2}\right)\right\}+\frac{\nabla \psi \cdot \nabla \theta}{\mathrm{R}^{2} \mathrm{~B}^{2}} \frac{\partial}{\partial \theta} \ln \mathrm{B}\right]\right\} \\
& +\mathrm{i} \frac{\mathrm{I}^{2}}{\omega q R^{2}} \sum_{\mathrm{j}} \int \mathrm{d}^{3} \mathrm{v} \frac{\mathrm{m}_{\mathrm{j}} \mathrm{v}_{\|}}{\mathrm{B}} \frac{\partial}{\partial \theta}\left(\frac{\mathrm{v}_{\|}}{\mathrm{B}}\right)\left[\frac{\mathrm{e}_{\mathrm{j}}}{\mathrm{T}_{\mathrm{j}}}\left(1-\frac{\omega_{* \mathrm{j}}^{\mathrm{T}}}{\omega}\right) \Psi^{(1)^{\prime}} \mathrm{f}_{0 \mathrm{j}}+\mathrm{h}_{\mathrm{j}}^{\prime}\right]-\frac{\mathrm{i}}{\omega} \sum_{\mathrm{j}} \frac{\mathrm{m}_{\mathrm{j}}}{\mathrm{e}_{\mathrm{j}}} \int \mathrm{d}^{3} \mathrm{v} \sigma \mid \mathrm{v}_{\|} \|_{\mathrm{j}} \mathbf{b} \cdot \nabla\left(\frac{\mathrm{m}_{\mathrm{j}} \mathrm{v}_{\perp}^{2}|\nabla \psi|^{2}}{2 \mathrm{~B}^{2}}\right) \mathrm{g}^{\prime \prime}
\end{aligned}
$$

Applying the annihilator \langle\rangle to eqn. (77) and using eqn. (76), we obtain

$$
\begin{aligned}
& \frac{\mathrm{I}^{2}}{\omega^{2}}\left[\frac{\mathrm{n}^{2} \mathrm{q}^{\prime 2}}{\mu_{0} \mathrm{q}^{2}}\left\langle\frac{\mathrm{B}^{2}}{|\nabla \psi|^{2}}\right\rangle^{-1}\left\langle\frac{1}{\mathrm{R}^{2}}\right\rangle^{2} \mathrm{x} \frac{\mathrm{d}^{2}\left(\mathrm{x} \Psi^{(0)}\right)}{\mathrm{dx} \mathrm{x}^{2}}+\frac{\mathrm{nq} \mathrm{q}^{\prime} \mathrm{x}}{\mathrm{q}} \omega \tilde{\mathrm{p}}^{\prime}\left(\left\langle\frac{1}{\mathrm{~B}^{2} \mathrm{R}^{2}}\right\rangle-\left\langle\frac{1}{\mathrm{R}^{2}}\right\rangle\left\langle\frac{1}{|\nabla \psi|^{2}}\right\rangle\left\langle\frac{\mathrm{B}^{2}}{|\nabla \psi|^{2}}\right\rangle^{-1}\right)\right] \\
& =m_{i} n_{i}\left\langle\frac{|\nabla \psi|^{2}}{B^{2}}\right\rangle\left[1-\frac{\omega_{r_{i}}}{\omega}\left(1+\eta_{i}\right)\right] \frac{d^{2} \Phi}{\mathrm{dx}^{2}}+\frac{\mathrm{np}_{0}^{\prime}}{\omega}\left\langle\frac{\tilde{\mathrm{B}}_{\|}}{\mathrm{B}}\right\rangle-\frac{\mathrm{n}}{\omega} \frac{\mathrm{np} \mathrm{p}_{0}^{\prime}}{\omega}\left\langle\frac{|\nabla \psi|^{2}}{\mathrm{R}^{2} \mathrm{~B}^{2}} \frac{\partial}{\partial \psi}\left(\mu_{0} \mathrm{p}+\mathrm{B}^{2}\right)+\frac{2 \nabla \psi \cdot \nabla \theta}{\mathrm{R}^{2} \mathrm{~B}^{2}} \frac{\partial}{\partial \theta} \ln \mathrm{B}\right\rangle \Psi^{(0)} \\
& -\frac{\mathrm{n}}{\omega}\left\langle\sum_{\mathrm{j}} \int^{3} \mathrm{~d}_{\mathrm{j}}\left[\frac{\mathrm{m}_{\mathrm{j}} \mathrm{v}_{\perp}^{2}}{2}\left(\frac{2|\nabla \psi|^{2}}{\mathrm{R}^{2} \mathrm{~B}^{2}} \frac{\partial}{\partial \psi}+\frac{\nabla \psi \cdot \nabla \theta}{\mathrm{R}^{2} \mathrm{~B}^{2}} \frac{\partial}{\partial \theta}\right) \ln \mathrm{B}+\mathrm{m}_{\mathrm{j}} \mathrm{v}_{\|}^{2}\left[\frac{2|\nabla \psi|^{2}}{\mathrm{R}^{2} \mathrm{~B}^{2}}\left\{\frac{1}{\mathrm{~B}^{4}} \frac{\partial}{\partial \psi}\left(\mu_{0} \mathrm{p}+\frac{\mathrm{B}^{2}}{2}\right)\right\}+\frac{\nabla \psi \cdot \nabla \theta}{\mathrm{R}^{2} \mathrm{~B}^{2}} \frac{\partial}{\partial \theta} \ln \mathrm{B}\right]\right]\right) \\
& -i \frac{I}{\omega}\left\langle\sum_{j} \int d^{3} v \frac{m_{j} v_{\|}}{B} \frac{I}{R^{2} q} \frac{\partial}{\partial \theta}\left(\frac{v_{\|}}{B}\right) h_{j}^{\prime}\right\rangle+\frac{i n I^{2}}{\omega^{2} q} p_{0}^{\prime}\left\langle\frac{1}{R^{2} B^{2}} \frac{\partial}{\partial \theta} \Psi^{(1)^{\prime}}\right\rangle
\end{aligned}
$$


where we have noted that $\Phi=\Phi(\mathrm{x})$ and the last term vanishes for an up-down symmetric equilibrium. Substituting for $\tilde{\mathrm{B}}_{\|}$from eqn. (56) and evaluating the velocity integrals over $h_{j 0}$ in the normal curvature term, the right-hand side of eqn. (78) becomes

$$
\begin{aligned}
& \mathrm{m}_{\mathrm{i}} \mathrm{n}_{\mathrm{i}}\left\langle\frac{|\nabla \psi|^{2}}{\mathrm{~B}^{2}}\right\rangle\left[1-\frac{\omega_{*_{\mathrm{i}}}}{\omega}\left(1+\eta_{\mathrm{i}}\right)\right] \frac{\mathrm{d}^{2} \Phi}{\mathrm{dx}^{2}}-\frac{2 \mathrm{n}}{\omega} \tilde{\mathrm{p}}\left\langle\frac{|\nabla \psi|^{2}}{\mathrm{R}^{2} \mathrm{~B}^{2}} \frac{\partial}{\partial \psi}\left(\mu_{0} \mathrm{p}+\frac{\mathrm{B}^{2}}{2}\right)+\frac{\nabla \psi \cdot \nabla \theta}{\mathrm{R}^{2} \mathrm{~B}^{2}} \frac{\partial}{\partial \theta} \ln \mathrm{B}\right\rangle \\
& -\mathrm{i} \frac{\mathrm{I}}{\omega}\left\langle\sum_{\mathrm{j}} \int \mathrm{d}^{3} \mathrm{v} \frac{\mathrm{m}_{\mathrm{j}} \mathrm{v}_{\|}}{\mathrm{B}} \frac{\mathrm{I}}{\mathrm{R}^{2} \mathrm{q}} \frac{\partial}{\partial \theta}\left(\frac{\mathrm{v}_{\|}}{\mathrm{B}}\right) \mathrm{h}_{\mathrm{j}}^{\prime}\right\rangle+\frac{\mathrm{inI}^{2}}{\omega^{2} \mathrm{q}} \mathrm{p}_{0}^{\prime}\left\langle\frac{1}{\mathrm{R}^{2} \mathrm{~B}^{2}} \frac{\partial}{\partial \theta} \Psi^{(1)^{\prime}}\right\rangle
\end{aligned}
$$

where we have expressed $\hat{p}$ in terms of $\tilde{p}$. Using eqn. (76) the last term can be evaluated:

$$
\begin{aligned}
& \frac{\mathrm{inI}^{2}}{\omega^{2} \mathrm{q} \mu_{0}}\left\langle\frac{1}{\mathrm{R}^{2} \mathrm{~B}^{2}} \frac{\partial}{\partial \theta} \Psi^{(1)^{\prime}}\right\rangle \mathrm{p}_{0}^{\prime}=-\frac{\mathrm{np}_{0}^{\prime} \mathrm{I}^{2}}{\omega} \tilde{\mathrm{p}}\left[\left\langle\frac{1}{|\nabla \psi|^{2} \mathrm{~B}^{2}}\right\rangle-\left\langle\frac{\mathrm{B}^{2}}{|\nabla \psi|^{2}}\right\rangle^{-1}\left\langle\frac{1}{|\nabla \psi|^{2}}\right\rangle^{2}\right] \\
& -\mathrm{p}_{0}^{\prime} \frac{\mathrm{n}^{2} \mathrm{q}^{\prime} \mathrm{I}^{2}}{\omega^{2} \mathrm{q}}\left(\mathrm{x} \Psi^{(0)}\right)^{\prime}\left[\left\langle\frac{1}{\mathrm{R}^{2}}\right\rangle\left\langle\frac{1}{|\nabla \psi|^{2}}\right\rangle\left\langle\frac{\mathrm{B}^{2}}{|\nabla \psi|^{2}}\right\rangle^{-1}-\left\langle\frac{1}{\mathrm{R}^{2} \mathrm{~B}^{2}}\right\rangle\right]
\end{aligned}
$$

To evaluate the penultimate term, we make repeated use of the gyro-kinetic equations for $\mathrm{h}_{\mathrm{j} 0}, \mathrm{~h}_{\mathrm{j} 1}, \mathrm{~h}_{\mathrm{j} 2}$ and $\mathrm{h}_{\mathrm{j} 3}$, with integrations by parts in $\theta$ and utilise the conservation of momentum in ion-ion collisions. Thus,

$$
-i \frac{I}{\omega}\left\langle\sum_{j} e_{j} \int d^{3} v \frac{I_{\|}}{R^{2} B q} \frac{\partial}{\partial \theta}\left(\frac{I_{\|}}{\Omega_{j}}\right) h_{j}^{\prime}\right\rangle=i \frac{I}{\omega}\left\langle\sum_{j} \int d^{3} v \frac{I_{\|} m_{j}}{B} \frac{I_{\|}}{R^{2} B q} \frac{\partial}{\partial \theta} h_{j}^{\prime}\right\rangle
$$

and using the gyro-kinetic equation (13) for $h_{j}$ we have

$$
\begin{aligned}
& i \frac{I}{\omega}\left\langle\sum_{j} \int d^{3} v \frac{I v_{\|} m_{j}}{B} \frac{I v_{\|}}{R^{2} B q} \frac{\partial}{\partial \theta} h_{j}^{\prime}\right\rangle= \\
& i \frac{I}{\omega}\left\langle\sum_{j} \int d^{3} v \frac{I_{\|} m_{j}}{B}\left\{-\frac{I_{\|}}{R^{2} B q} \frac{\partial}{\partial \theta}\left(\frac{I v_{\|}}{B}\right) h_{j}^{\prime \prime}+C_{j}\left(h_{j}^{\prime}\right)+i \omega h_{j}^{\prime}-i n q^{\prime} \frac{I_{\|}}{R^{2} B q}\left(x h_{j}\right)^{\prime}\right\}\right\rangle
\end{aligned}
$$

since the right-hand side driving term in eqn. (13) is of even parity in $\mathrm{v}_{\|}$. 
We substitute the lowest order in $\mathrm{h}_{\mathrm{j}}$ that gives a non-vanishing contribution to the various terms. For the last term this is $h_{j o}$, leading to a term related to the perturbed pressure $\tilde{\mathrm{p}}_{\mathrm{j}}$ while for the penultimate term, it is $\mathrm{h}_{\mathrm{j} 1}$, leading to a term related to the perturbed parallel momentum, $\mathrm{m}_{\mathrm{j}} \mathrm{u}_{\| \mathrm{j}}$; the latter is clearly dominated by the heavier ions. The collisional term vanishes due to momentum conservation. The first term can clearly be recognised as related to the radial flux of toroidal momentum, $\Pi$ :

$$
\left\langle\frac{\Pi}{B}\right\rangle=\left\langle\sum_{j} \int d^{3} v \frac{m_{j} v_{\|}}{B} v_{d r j} h_{j}\right\rangle
$$

The contributions to this term from $h_{j 1}$ and $h_{j 2}$ vanish on integration by parts in $\theta$ and the first non-vanishing contribution is from $h_{j 3}$ and is also dominated by the ions. Thus expression (82) reduces to

$$
-i \frac{I}{\omega}\left\langle\frac{\Pi}{B}\right\rangle^{\prime \prime}-\operatorname{Im}_{i} n_{i}\left\langle\frac{u_{\| i}}{B}\right\rangle^{\prime}+\frac{n q^{\prime} I^{2}}{\omega q}\left\langle\frac{1}{R^{2} B^{2}}\right\rangle(x \hat{p})^{\prime}
$$

To calculate the term involving $\Pi\left(\mathrm{h}_{\mathrm{j} 3}\right)$ we again integrate by parts in $\theta$ and use the gyrokinetic equation for $h_{j 3}$ to obtain

$$
-i\left\langle\frac{\Pi}{B}\right\rangle^{\prime \prime}=i \frac{I}{2 \omega}\left\langle\sum_{j} \int d^{3} v \frac{m_{j} v_{\|}}{B} \frac{I v_{\|}}{\Omega_{j}}\left\{-\frac{I_{\|}}{R^{2} B q} \frac{\partial}{\partial \theta}\left(\frac{I v_{\|}}{\Omega_{j}}\right) h_{j 2}^{\prime \prime \prime}+C_{j}\left(h_{j 2}^{\prime \prime}\right)\right\}\right.
$$

where the omitted terms are of higher order than those retained previously in eqn. (84). In a similar manner a further integration by parts on the first term and use of the gyrokinetic equation yields

$$
-\frac{i}{\omega}\left\langle\frac{\Pi}{B}\right\rangle^{\prime \prime}=i \frac{I}{2 \omega}\left\langle\sum_{j} \int d^{3} v \frac{m_{j} v_{\|}}{B}\left\{C_{j}\left(h_{j 2}^{\prime \prime}\right)-\frac{I_{\|}}{3 \Omega_{j}} C_{j}\left(h_{j 1}^{\prime \prime \prime}\right)\right\}\right\rangle
$$

This requires calculation of $\mathrm{h}_{\mathrm{j} 2}$; such a calculation was carried out in Ref. 18, but a later more elegant treatment using the adjoint function to $\mathrm{h}_{\mathrm{j} 1}$ [19], identified a numerical error in Ref. 18. Both treatments exploited an approximate 'similarity' between the constraint equations on $h_{j 1}$ and $h_{j 2}$ and the integrals required for evaluating the quantity $\Pi$; this similarity requires a weak poloidal variation of $\mathrm{B}(\theta)$. Although the accuracy of these 
calculations is not entirely clear, we propose to identify $\Pi \equiv \Pi_{2}$ where $\Pi_{2}$ is defined in eqn. (5) of Ref. 19. Using the evaluation of $\Pi_{2}$ given in eqn. (33) of Ref. 19, we find

$$
\Pi=0.1862 \mathrm{~m}_{\mathrm{i}} \mathrm{n}_{\mathrm{i}}(2 \hat{\varepsilon})^{3 / 2}\langle\mathrm{R}\rangle^{2} \frac{\mathrm{m}_{\mathrm{i}} \mathrm{T}_{\mathrm{i}}}{\tau_{\mathrm{i}} \mathrm{e}^{3}} \tilde{\mathrm{T}}_{\mathrm{i}}^{\prime \prime}
$$

where $\hat{\varepsilon}=\left(R_{\max }-R_{\min }\right) / 2\langle R\rangle$ on a flux surface.

Assembling all the contributions to eqn. (78) we have

$$
\begin{aligned}
& \left(\frac{\mathrm{q}^{\prime}}{\mathrm{q}}\right)^{2} \frac{1}{\mu_{0}}\left\langle\frac{\mathrm{I}}{\mathrm{R}^{2}}\right\rangle^{2} \mathrm{x} \frac{\mathrm{d}^{2}}{\mathrm{dx^{2 }}}\left(\mathrm{x} \Psi^{(0)}\right)+\mathrm{x}\left(\frac{\mathrm{q}^{\prime}}{\mathrm{q}}\right)\left[\left\langle\frac{\mathrm{I}^{2}}{\mathrm{R}^{2} \mathrm{~B}^{2}}\right\rangle\left\langle\frac{\mathrm{B}^{2}}{|\nabla \psi|^{2}}\right\rangle-\left\langle\frac{\mathrm{I}^{2}}{\mathrm{R}^{2}}\right\rangle\left\langle\frac{1}{|\nabla \psi|^{2}}\right\rangle\right] \frac{\omega}{\mathrm{n}} \tilde{\mathrm{p}}^{\prime} \\
& +\mu_{0} \mathrm{p}_{0}^{\prime}\left[\left\langle\frac{\mathrm{I}^{2}}{|\nabla \psi|^{2} \mathrm{~B}^{2}}\right\rangle\left\langle\frac{\mathrm{B}^{2}}{|\nabla \psi|^{2}}\right\rangle-\left\langle\frac{\mathrm{I}}{|\nabla \psi|^{2}}\right\rangle^{2}\right] \frac{\omega}{\mathrm{n}} \tilde{\mathrm{p}}+\mathrm{p}_{0}^{\prime} \frac{\mathrm{q}^{\prime}}{\mathrm{q}}\left[\left\langle\frac{\mathrm{I}^{2}}{\mathrm{R}^{2}}\right\rangle\left\langle\frac{1}{|\nabla \psi|^{2}}\right\rangle-\left\langle\frac{\mathrm{B}^{2}}{|\nabla \psi|^{2}}\right\rangle\left\langle\frac{\mathrm{I}^{2}}{\mathrm{R}^{2} \mathrm{~B}^{2}}\right\rangle\right]\left(\mathrm{x}^{(0)}\right)^{\prime} \\
& +2\left\langle\frac{|\nabla \psi|^{2}}{\mathrm{R}^{2} \mathrm{~B}^{4}} \frac{\partial}{\partial \psi}\left(\mu_{0} \mathrm{p}+\frac{\mathrm{B}^{2}}{2}\right)+\frac{\nabla \psi \cdot \nabla \theta}{\mathrm{R}^{2} \mathrm{~B}^{4}} \frac{\partial}{\partial \theta}\left(\frac{\mathrm{B}^{2}}{2}\right)\right\rangle\left\langle\frac{\mathrm{B}^{2}}{|\nabla \psi|^{2}}\right| \frac{\omega}{\mathrm{n}} \tilde{\mathrm{p}}-\frac{\mathrm{q}^{\prime}}{\mathrm{q}}\left\langle\frac{\mathrm{I}^{2}}{\mathrm{R}^{2} \mathrm{~B}^{2}}\right\rangle\left\langle\frac{\mathrm{B}^{2}}{|\nabla \psi|^{2}}\right\rangle \frac{\omega \mathrm{x}}{\mathrm{n}}(\mathrm{x} \hat{\mathrm{p}})^{\prime}+\frac{\mathrm{i} \omega}{\mathrm{n}^{2}} \Pi^{\prime \prime}\left\langle\frac{\mathrm{B}^{2}}{|\nabla \psi|^{2}}\right\rangle \\
& =\frac{\mathrm{m}_{\mathrm{i}} \mathrm{n}_{\mathrm{i}}}{\mathrm{n}^{2}}\left\langle\frac{\mathrm{B}^{2}}{|\nabla \psi|^{2}}\right\rangle\left[\left\langle\frac{|\nabla \psi|^{2}}{\mathrm{~B}^{2}}\right\rangle \omega\left(\omega-\omega_{*_{\mathrm{i}}}\left(1+\eta_{\mathrm{i}}\right)\right) \frac{\mathrm{d}^{2} \Phi}{\mathrm{dx}^{2}}-\omega^{2}\left\langle\frac{\mathrm{Iu} \| \mathrm{i}}{\mathrm{B}}\right\rangle\right]
\end{aligned}
$$

Substituting for $\mathrm{u}_{\| \mathrm{i}}$ from eqn. (49) we can write the inertial term on the right-hand side of eqn. (88) as

$$
\begin{aligned}
& \frac{m_{i} n_{i}}{n^{2}}\left\langle\frac{B^{2}}{|\nabla \psi|^{2}}\right\rangle\left[\omega\left(\omega-\omega_{*_{i}}\left(1+\eta_{i}\right)\right)\left(\left\langle\frac{|\nabla \psi|^{2}}{B^{2}}\right\rangle \frac{d^{2} \Phi}{d x^{2}}+\left\langle\frac{I^{2}}{B^{2}}\right\rangle \frac{d^{2} \Psi^{(0)}}{d x^{2}}\right)-1.17 n \omega T_{i 0}^{\prime} \frac{I^{2} f_{c}}{\left\langle B^{2}\right\rangle} \frac{d^{2} \Psi^{(0)}}{d x^{2}}\right] \\
& +\frac{m_{i} n_{i}}{n^{2}}\left\langle\frac{B^{2}}{|\nabla \psi|^{2}}\right\rangle\left[\omega^{2}\left\langle\frac{I^{2}}{B^{2}}\right\rangle \frac{\hat{p}_{i}^{\prime \prime}}{\mathrm{en}_{i}}-1.17 \omega^{2} \frac{I^{2} f_{c}}{\left\langle B^{2}\right\rangle} \frac{\hat{T}_{i}^{\prime \prime}}{e}\right]
\end{aligned}
$$

Here one can recognise the neoclassical enhancement of the ion inertia when one sets $\Psi^{(0)}=\Phi$. The cross field momentum transport term, $\Pi$, in eqn. (88) is given by eqn. (87) in terms of $\hat{T}_{i} \cdot \hat{T}_{i}$, which also appears in the inertial term, is itself given by eqn. (53). 
It is interesting to separate out the convective parts of $\tilde{p}$ proportional to $\Psi$ in eqn. (57). The terms in $\Psi^{\prime}, \Psi, \hat{p}^{\prime}$ and $\hat{\mathrm{p}}$ can then be combined and expressed in terms of the fluxsurface-averaged quantities, E, F, and H defined by Glasser et al. [13, 20] and L defined in Ref. 5 (see Appendix A):

$$
\begin{aligned}
& x \frac{d^{2}}{d x^{2}}\left(x \Psi^{(0)}\right)+D_{I} \Psi^{(0)}-(\omega x \hat{p} / n)^{\prime}\left((L+H) / p_{0}^{\prime}\right)+D_{I}\left(\omega \hat{p} / n p_{0}^{\prime}\right) \\
& =\frac{\mu_{0} m_{i} n_{i}}{n^{2}}\left(\frac{\mathrm{q}}{\mathrm{Iq}^{\prime}\left\langle 1 / \mathrm{R}^{2}\right\rangle}\right)^{2}\left\langle\frac{\mathrm{B}^{2}}{|\nabla \psi|^{2}}\right\rangle\left[\omega\left(\omega-\omega_{*_{\mathrm{i}}}\left(1+\eta_{\mathrm{i}}\right)\right)\left(\left\langle\frac{|\nabla \psi|^{2}}{\mathrm{~B}^{2}}\right) \frac{\mathrm{d}^{2} \Phi}{\mathrm{dx}}+\left\langle\frac{\mathrm{I}^{2}}{\mathrm{~B}^{2}}\right\rangle \frac{\mathrm{d}^{2} \Psi^{(0)}}{\mathrm{dx}^{2}}\right)-1.17 \mathrm{n} \omega \mathrm{T}_{\mathrm{i} 0}^{\prime} \frac{\mathrm{I}^{2} \mathrm{f}_{\mathrm{c}}}{\left\langle\mathrm{B}^{2}\right\rangle} \frac{\mathrm{d}^{2} \Psi^{(0)}}{\mathrm{dx} \mathrm{x}^{2}}\right] \\
& +\frac{\mu_{0} \mathrm{~m}_{\mathrm{i}} \mathrm{n}_{\mathrm{i}}}{\mathrm{n}^{2}}\left(\frac{\mathrm{q}}{\mathrm{Iq}\left\langle\mathrm{q}^{\prime} / \mathrm{R}^{2}\right\rangle}\right)^{2}\left\langle\frac{\mathrm{B}^{2}}{|\nabla \psi|^{2}}\right\rangle\left[\omega^{2}\left\langle\frac{\mathrm{I}^{2}}{\mathrm{~B}^{2}}\right\rangle \frac{\hat{\mathrm{p}}_{\mathrm{i}}^{\prime \prime}}{\mathrm{en}_{\mathrm{i}}}-1.17 \omega^{2} \frac{\mathrm{I}^{2} \mathrm{f}_{\mathrm{c}}}{\left\langle\mathrm{B}^{2}\right\rangle} \frac{\hat{\mathrm{T}}_{\mathrm{i}}^{\prime \prime}}{\mathrm{e}}\right]-\frac{\mathrm{i} \omega \mu_{0}}{\mathrm{n}^{2}} \Pi^{\prime \prime}\left(\frac{\mathrm{q}}{\mathrm{Iq}^{\prime}\left\langle 1 / \mathrm{R}^{2}\right\rangle}\right)^{2}\left\langle\frac{\mathrm{B}^{2}}{|\nabla \psi|^{2}}\right\rangle
\end{aligned}
$$

where $\mathrm{D}_{\mathrm{I}}=\mathrm{E}+\mathrm{F}+\mathrm{H}$.

We can simplify eqn. (90) further if we ignore neoclassical ion thermal and transport and viscosity. Using relations (52) and (53) to determine $\hat{\mathrm{p}}_{\mathrm{i}}$ and $\hat{\mathrm{T}}_{\mathrm{i}}$ in the inertial term on the right-hand side of eqn. (90) (ignoring contributions from $\tilde{\mathrm{B}}_{\|}$), we obtain:

$$
\begin{aligned}
& x\left(d^{2}\left(x \Psi^{(0)}\right) / d x^{2}\right)+D_{I} \Psi^{(0)}-\left(\omega / n p_{0}^{\prime}\right)(x \hat{p})^{\prime}(\mathrm{L}+\mathrm{H})+D_{\mathrm{I}}\left(\omega \hat{\mathrm{p}} / \mathrm{np}_{0}^{\prime}\right)= \\
& \left(\mu_{0} \mathrm{~m}_{\mathrm{i}} \mathrm{n}_{\mathrm{i}} \omega^{2} / \mathrm{n}^{2}\right)\left(\mathrm{q} / \mathrm{Iq}^{\prime}\left\langle 1 / \mathrm{R}^{2}\right\rangle\right)^{2}\left\langle\mathrm{~B}^{2} /|\nabla \psi|^{2}\right\rangle\left\langle\left\langle\mathrm{R}^{2}\right\rangle\left(1-\omega_{* \mathrm{pi}} / \omega\right)+\left(1.17 \mathrm{f}_{\mathrm{c}} \mathrm{I}^{2} /\left\langle\mathrm{B}^{2}\right\rangle\right) \omega_{*_{\mathrm{pi}}} \eta_{\mathrm{i}} / \omega\right\}\left(\mathrm{d}^{2} \Phi^{(0)} / \mathrm{dx}^{2}\right)
\end{aligned}
$$

The contributions from $\hat{\mathrm{p}}$, determined by eqns. (38), (39) and (52), have introduced effects from neoclassical ion compressibility through L; this equation also includes the effects of ion neoclassical inertia

\section{$5 \quad$ Eigenvalue problem}

The complete set of equations determining the stability of the system consists of the vorticity equation (90) with expression (87) for the perpendicular viscosity, $\Pi$ and Ohm's law (68). However, these equations also involve the perturbed pressures, densities and temperatures given in eqns. (37), ignoring the neoclassical transport, (38) and (39) for electrons and eqns. (51), (52) and (53) for ions. Expression (56) provides the perturbed magnetic field, $\tilde{\mathrm{B}}_{\|}$, but this is largely unimportant for normal values of $\beta$. Thus the general eigenvalue problem obtained by this method has the form of a tenth order 
system of ODE's, if we include cross-field neoclassical electron transport; ignoring this, as is very reasonable, it reduces to an eighth order system. If we also ignore ion neoclassical thermal transport and viscosity as in eqn. (91), then it simplifies to a fourth order system.

\section{(a) The Collisional Case}

However it is interesting to take the collisional limit, $s \rightarrow 0$, since this generalises Refs. 2 and 5 to include the effect of temperature gradients. Then the expressions for $\hat{n}_{j}$ and $\hat{T}_{j}$ are simpler and Fourier transforming allows one to reduce the Ohm's law and vorticity equations to a single second order ODE. This differs from the results in Refs. 2 and 5 by the substitution:

$$
\omega-\omega_{*_{\mathrm{e}}} \rightarrow \omega-\omega_{*_{\mathrm{e}}}\left[1+\left(5.9-1.7 \mathrm{f}_{\mathrm{c}}\right) \eta_{\mathrm{e}} /\left(2.45-0.45 \mathrm{f}_{\mathrm{c}}\right)\right]
$$

in Ohm's law (68) and the substitution

$$
\left(1-\omega_{*_{\mathrm{i}}} / \omega\right) \rightarrow\left\{\left(1-\omega_{*_{\mathrm{pi}}} / \omega\right)+\left(1.17 \mathrm{f}_{\mathrm{c}} \mathrm{I}^{2} /\langle\mathrm{B}\rangle^{2}\langle\mathrm{R}\rangle^{2}\right) \omega_{*_{\mathrm{pi}}} \eta_{\mathrm{i}} / \omega\right\}
$$

in the inertia term in the vorticity equation (91).

\section{(b) The Semi-collisional Case}

The semi-collisional regime is more appropriate to JET or ITER-like conditions than the collisional case discussed in the previous sub-section. This corresponds to assuming the semi-collisional quantity $\mathrm{s} \sim \mathrm{O}(1)$, rather than $\mathrm{s}<<1$, in the resonant layer. To simplify the analysis we ignore the curvature terms, $\mathrm{D}_{\mathrm{I}}$ and $\mathrm{H}$, although we retain the neoclassical effects represented by $L$ and $f_{t}$. We proceed by analogy with the approach to analysing the semi-collisional regime adopted by Drake et al. [8], although our treatment is complicated by the presence of the bootstrap current in Ohm's law and neoclassical compressibility effects in the vorticity equation. With these assumptions we can reorganise the vorticity equation (91) and Ampère's law (68) with the neoclassical current as follows. On using Ampère's law, the vorticity equation becomes:

$$
\begin{aligned}
& {\left[1+\frac{1+\eta_{i}}{\hat{\omega} \tau}-1.17 f_{c} \frac{I^{2}}{\left\langle R^{2}\right\rangle\left\langle B^{2}\right\rangle} \frac{\eta_{i}}{\hat{\omega} \tau}\right] \frac{d^{2} \Phi}{d s^{2}}=} \\
& -i C\left\{s^{2} \hat{\sigma}\left(s^{2}\right)(\Phi-\Psi)+\frac{T_{e}}{e} \frac{\hat{\gamma}}{\hat{\beta}} s\left[\alpha_{n}(1+1 / \tau) \frac{d}{d s}\left(\frac{\tilde{n}_{e}}{n_{0}}\right)+\alpha_{e} \frac{d}{d s}\left(\frac{\tilde{T}_{e}}{T_{e}}\right)+\alpha_{1} \frac{d}{d s}\left(\frac{\tilde{T}_{i}}{T_{e}}\right)\right]-\frac{T_{e}}{e} \frac{\hat{\mu}}{\hat{\beta}} \frac{d}{d s}\left(\frac{s \hat{p}}{p_{e}}\right)\right\}
\end{aligned}
$$

while Ampère's law takes the form: 


$$
\frac{\mathrm{d}^{2}}{\mathrm{ds}^{2}}(\mathrm{~s} \Psi)=\hat{\omega}\left\{\hat{\beta} s \hat{\sigma}\left(\mathrm{s}^{2}\right)(\Phi-\Psi)+\frac{\mathrm{T}_{\mathrm{e}}}{\mathrm{e}} \hat{\gamma}\left[\alpha_{\mathrm{n}}(1+1 / \tau) \frac{\mathrm{d}}{\mathrm{ds}}\left(\frac{\tilde{\mathrm{n}}_{\mathrm{e}}}{\mathrm{n}_{0}}\right)+\alpha_{\mathrm{e}} \frac{\mathrm{d}}{\mathrm{ds}}\left(\frac{\tilde{\mathrm{T}}_{\mathrm{e}}}{\mathrm{T}_{\mathrm{e} 0}}\right)+\alpha_{\mathrm{i}} \frac{\mathrm{d}}{\mathrm{ds}}\left(\frac{\tilde{\mathrm{T}}_{\mathrm{i}}}{\mathrm{T}_{\mathrm{e} 0}}\right)\right]\right\}
$$

Here we have defined

$$
\begin{gathered}
\hat{\beta}=\mu_{0} \mathrm{p}_{\mathrm{e} 0}\left(\frac{\mathrm{d}\left(\ln \mathrm{n}_{\mathrm{e} 0}\right)}{\mathrm{d}(\ell \mathrm{nq})}\right)^{2} \frac{\left\langle\mathrm{B}^{2} /|\nabla \psi|^{2}\right\rangle}{\mathrm{I}^{2}\left\langle 1 / \mathrm{R}^{2}\right\rangle^{2}}, \hat{\gamma}=\mu_{0} \mathrm{p}_{\mathrm{e} 0} \frac{\left(1-\mathrm{f}_{\mathrm{c}}\right)}{\left(1-0.37 \mathrm{f}_{\mathrm{c}}\right)}\left(\frac{\mathrm{d}\left(\ln \mathrm{n}_{\mathrm{e} 0}\right)}{\mathrm{d}(\ell \mathrm{nq})}\right) \frac{\left\langle\mathrm{B}^{2} /|\nabla \psi|^{2}\right\rangle}{\left\langle\mathrm{B}^{2}\right\rangle\left\langle 1 / \mathrm{R}^{2}\right\rangle} \\
\hat{\mu}=\mu_{0} \mathrm{p}_{\mathrm{e} 0}\left(\frac{\mathrm{d}\left(\ln \mathrm{n}_{\mathrm{e} 0}\right)}{\mathrm{d}(\ell \mathrm{nq})}\right) \frac{\left\langle\mathrm{B}^{2} /|\nabla \psi|^{2}\right\rangle}{\left\langle\mathrm{B}^{2}\right\rangle\left\langle 1 / \mathrm{R}^{2}\right\rangle}, \mathrm{C}=\left(\frac{\mathrm{m}_{\mathrm{e}}}{\mathrm{m}_{\mathrm{i}}}\right)\left(\frac{\mathrm{v}_{0}}{\omega_{\mathrm{e}}}\right) \frac{\left(1-0.37 \mathrm{f}_{\mathrm{c}}\right)\left\langle\mathrm{B}^{2}\right\rangle}{\mathrm{f}_{\mathrm{c}}\left\langle\mathrm{I}^{2} / \mathrm{R}^{2}\right\rangle\left\langle\mathrm{R}^{2}\right\rangle\left\langle 1 / \mathrm{R}^{2}\right\rangle}\left(\frac{\mathrm{d}\left(\ln \mathrm{n}_{\mathrm{e} 0}\right)}{\mathrm{d}(\ln \mathrm{q})}\right)^{2} \\
\hat{\sigma}\left(\mathrm{s}^{2}\right)=\left\{(\hat{\omega}-1)\left(\sigma_{\mathrm{n}}+\mathrm{d}_{1} \mathrm{~s}^{2}\right)-\eta_{\mathrm{e}} \sigma_{\mathrm{T}}\right\} / \mathrm{D} \equiv\left(\sigma_{0}+\sigma_{\mathrm{l}} \mathrm{s}^{2}\right) / \mathrm{D} ; \\
\hat{\omega}=\omega / \omega_{*_{\mathrm{e}}}, \tau=\mathrm{T}_{\mathrm{e} 0} / \mathrm{T}_{\mathrm{i} 0}
\end{gathered}
$$

Note that, for normal density profiles, both $\hat{\gamma}<0$ and $\hat{\mu}<0$. The expression for $\hat{\beta}$ reduces to that of Ref. 8 in the cylindrical limit, while $\hat{\gamma}$ and $\hat{\mu}$ arise entirely from toroidal neoclassical effects: $\hat{\gamma}$ from the bootstrap current and $\hat{\mu}$ from neoclassical compressibility effects. While $\mathrm{C}$ resembles the collisionality parameter in Ref. 8, it is reduced by a factor $\sim\left(\mathrm{B}_{\varphi}^{2} / \mathrm{B}_{\theta}^{2}\right)$ as a result of the neoclassical enhancement in ion inertia.

\section{Discussion and Conclusions}

We have derived a set of equations that describe the linear stability of tearing modes in the low collisionality regime appropriate to large tokamaks such as JET or ITER. Although these have the form of fluid-like equations for moments such as plasma density, temperature and current to feed into Maxwell's equations, they contain coefficients that encapsulate kinetic neoclassical effects, such as cross-field transport of particles, energy and momentum, the bootstrap current, neoclassical resistivity and neoclassical ion inertia and compressibility. The electron model corresponds to the semi-collisional regime in 
which parallel diffusive transport effects compete with the mode frequency $\left(\omega \sim \mathrm{k}_{\|}^{2} \mathrm{v}_{\text {the }}^{2} / v_{\mathrm{e}}\right)$. Thus, using the definition of the electron layer width, $\delta_{\mathrm{sc}}$, from the semi-collisional theory, assuming $\omega \sim \omega_{*_{\mathrm{e}}}$, i.e. $\omega_{\mathrm{x}_{\mathrm{e}}} \sim\left(\mathrm{k}_{\theta} \delta_{\mathrm{sc}} \hat{\mathrm{s}} / \mathrm{Rq}\right)^{2} v_{\text {the }}^{2} / v_{\mathrm{e}}$, one finds

$$
\delta_{\mathrm{sc}} \sim\left(\frac{v_{\mathrm{e}}}{v_{\text {the }}} \frac{\mathrm{L}_{\mathrm{s}}^{2}}{\mathrm{~L}_{\mathrm{n}}} \frac{\mathrm{r} \rho_{\mathrm{e}}}{\mathrm{nq}}\right)^{1 / 2} \sim\left(\frac{\mathrm{a}}{\mathrm{R}}\right)^{3 / 4}\left(\frac{v_{\mathrm{*}_{\mathrm{e}}}}{\hat{\mathrm{s}}^{2}} \frac{\mathrm{Rq}}{\mathrm{L}_{\mathrm{n}}} \frac{\mathrm{r} \rho_{\mathrm{e}}}{\mathrm{nq}}\right)^{1 / 2}
$$

where $\mathrm{L}_{\mathrm{s}}=\mathrm{Rq} / \hat{\mathrm{s}}$ is the magnetic shear length. However, the ion model we use assumes that $\left(\mathrm{k}_{\mathrm{r}} \rho_{\mathrm{i}}\right)^{2} \sim\left(\rho_{\mathrm{i}} / \delta_{\mathrm{sc}}\right)^{2}<1$, but this can only be justified at low magnetic shear, $\hat{\mathrm{s}}$, or for a cold ion model. Using the definition (97) for the electron layer width, $\delta_{\mathrm{sc}}$, this implies

$$
\hat{\mathrm{s}}<\left(\frac{\mathrm{a}}{\mathrm{R}}\right)^{3 / 4}\left(\sqrt{\frac{\mathrm{m}_{\mathrm{e}}}{\mathrm{m}_{\mathrm{i}}}} \frac{\mathrm{Rq}}{\mathrm{L}_{\mathrm{n}}} \frac{\mathrm{v}_{*_{\mathrm{e}}}}{\rho_{*_{\mathrm{i}}}}\right)^{1 / 2} .
$$

Nevertheless this is highly relevant for describing the resistive internal kink mode involved in the sawtooth phenomenon. A treatment for finite shear requires a kinetic, large orbit theory $\left(\rho_{\mathrm{i}}>\delta_{\mathrm{sc}}\right)$, rather than the present fluid theory based on an ion Larmor radius expansion. This will be a major development, extending the cylindrical geometry theory of Cowley et al. [11] to the toroidal situation with finite ion banana orbits.

The full set of equations defining the eigenvalue problem was described at the beginning of Section 5: the vorticity equation (90) with expression (87) for the perpendicular viscosity, $\Pi$ (or, ignoring ion thermal transport and perpendicular viscosity, eqn. (91)), and Ohm's law (68). The perturbed pressures, densities and temperatures appearing in these equations are given by eqns. (38) and (39) for electrons and eqns. (51) and (53) for ions (one should note the relations (55), (57) and (65) between various perturbed quantities); eqn. (56) provides the perturbed magnetic field, $\tilde{\mathrm{B}}_{\|}$(unimportant for typical values of plasma pressure).

This system of equations is comprised of ordinary differential equations in a local 'radial' co-ordinate about the mode resonant surface, $\mathrm{m}=\mathrm{nq}(\mathrm{r})$. These can be of rather high order, but can be considerably simplified if we ignore the small electron neoclassical transport of density and temperature, i.e. $\omega>\varepsilon^{-3 / 2} q^{2} v_{\mathrm{e}} \rho_{\mathrm{e}}^{2}$. This leads to algebraic expressions for $\hat{n}_{e}$ and $\hat{T}_{e}$. A further simplification, plausible but less convincing, is to ignore ion neoclassical transport of energy and momentum when one can also have algebraic solutions for $\hat{n}_{i}$ and $\hat{T}_{i}$. The radial component of Maxwell's equation provides an algebraic expression for $\tilde{\mathrm{B}}_{\|}$; for normal values of plasma $\beta$ this can be ignored. With all these assumptions the system reduces to a fourth order set of differential equations, consisting of a neoclassical Ohm's law and the vorticity equation; in general this will 
require numerical solution, with boundary conditions determined by matching to outer solutions and therefore involving the tearing mode stability parameter $\Delta^{\prime}$.

One can identify two other characteristic lengths besides the semi-collisional layer width: the resistive layer width,

$$
\delta_{\eta} \sim\left(\frac{\eta}{\omega \mu_{0}}\right)^{1 / 2} \sim\left(\frac{L_{n}}{v_{\text {the }} \beta_{\mathrm{e}}} \frac{\rho_{\mathrm{e}} \mathrm{r}}{\mathrm{nq}}\right)^{1 / 2} \sim\left(\frac{\mathrm{a}}{\mathrm{R}}\right)^{3 / 4}\left(\frac{v_{\mathrm{*}_{\mathrm{e}}}}{\beta_{\mathrm{e}}} \frac{\mathrm{L}_{\mathrm{n}}}{\mathrm{Rq}} \frac{\rho_{\mathrm{e}} \mathrm{r}}{\mathrm{nq}}\right)^{1 / 2}
$$

and the ion neoclassical transport length scale (we can safely ignore the electron neoclassical transport scale)

$$
\delta_{x_{\mathrm{i}}} \sim\left(\frac{\mathrm{R}}{\mathrm{a}}\right)^{3 / 4}\left(\frac{v_{\mathrm{i}} \rho_{\mathrm{i}}{ }^{2} \mathrm{q}^{2}}{\omega}\right)^{1 / 2} \sim\left(v_{*_{\mathrm{i}}} \frac{\mathrm{L}_{\mathrm{n}}}{\mathrm{R}} \frac{\rho_{\mathrm{i}} \mathrm{r}}{\mathrm{n}}\right)^{1 / 2}
$$

Clearly, depending on parameters, all these can compete: e.g. the semi-collisional layer, where the electron responses $\left(\hat{p}_{e}, \hat{n}_{e}\right.$ and $\left.\hat{T}_{e}\right)$ have structure, can be broader or narrower than the resistive reconnection layer. One expects the collisional model to pertain if $\delta_{\eta}>\delta_{\mathrm{sc}}$; in fact, using the estimates (97) and (99), the ratio

$$
\left(\frac{\delta_{\mathrm{sc}}}{\delta_{\eta}}\right)^{2} \sim \frac{\beta_{\mathrm{e}}}{2}\left(\frac{\mathrm{L}_{\mathrm{s}}}{\mathrm{L}_{\mathrm{n}}}\right)^{2} \sim \frac{\beta_{\mathrm{e}}}{2 \mathrm{~s}^{2}}\left(\frac{\mathrm{Rq}}{\mathrm{L}_{\mathrm{n}}}\right)^{2}
$$

(see also Ref. 8) is likely to be $\mathrm{O}(1)$ for a sawtooth with $\hat{\mathrm{s}} \sim 0.1$, so one must indeed consider semi-collisional effects when modelling the sawtooth instability. Similarly one must consider the role of the ion responses $\left(\hat{\mathrm{p}}_{\mathrm{i}}, \hat{\mathrm{n}}_{\mathrm{i}}\right.$ and $\left.\hat{\mathrm{T}}_{\mathrm{i}}\right)$ due to ion neoclassical transport.

We have generalised the existing theory of linear neoclassical tearing modes in the collisional regime [2] to include the effects of temperature gradients. It is worth recalling that Ref. 2 identified a strong reduction in growth rate of the $\Delta^{\prime}$ driven tearing mode due to neoclassical resistivity and ion inertia effects, although the bootstrap current drive in the resonant layer would overwhelm the Glasser stabilisation effect [13] and lead to an unstable tearing parity 'interchange' mode, a linear analogue of finite island neoclassical tearing modes $[3,4]$. In the more relevant semi-collisional regime we have derived a pair of second-order differential equations, extending the work of Drake et al. [8] (an analytic solution of these will appear in a later paper.) These equations are modified by the effects of the bootstrap current, neoclassical resistivity, the neoclassical ion inertia (which reduces the effective collisionality parameter $\mathrm{C}$ in Ref. 8 by a factor $\left.\sim\left(\mathrm{B}_{\varphi}^{2} / \mathrm{B}_{\theta}^{2}\right)\right)$, as well as a neoclassical compression of the pressure response. In future one can also consider the numerical solution of these equations in the context of the low shear sawtooth situation, as well as an extension to include finite ion orbit effects for $\mathrm{m}>1$ tearing 
modes. Of course the theory can be applied to resistive ballooning modes and it considerably extends the earlier treatment of Refs. 2 and 5 by including more physics: thermal effects and semi-collisional effects. These techniques may also be useful in analysing the finite island theory of neoclassical tearing modes.

\section{Acknowledgements}

This work was partly funded by the United Kingdom Engineering and Physical Sciences Research Council and by the European Communities under the contract of Association between EURATOM and UKAEA. The views and opinions expressed herein do not necessarily reflect those of the European Commission.

\section{References}

[1] J D Callen and K C Shaing, Phys. Fluids 281845 (1985)

[2] T S Hahm, Phys. Fluids 313709 (1988)

[3] R Carrera, R D Hazeltine and M Kotchenreuther Phys. Fluids 29899 (1986)

[4] J D Callen, W X Qu, D Siebert, B A Carreras, K C Shaing and D A Spong, in Plasma Physics and Controlled Nuclear Fusion Research, 1986 (IAEA Vienna, 1987), Vol. II, p 157

[5] J W Connor and Liu Chen, Phys. Fluids 282201 (1985)

[6] J F Drake and Y C Lee, Phys. Fluids 201347 (1977)

[7] C S Chang, R R Dominguez and R D Hazeltine, Phys Fluids 241655 (1981)

[8] J F Drake, T M Antonsen Jr, A B Hassam and N T Gladd, Phys. Fluids 262509 (1983)

[9] B Coppi, J W-K Mark, L Sugiyama and G Bertin, Phys. Rev. Lett. 421058 (1979)

[10] P Savrukhin et al., Plasma Physics Control. Fusion 331347 (1991)

[11] S C Cowley, R M Kulsrud and T S Hahm, Phys. Fluids 293230 (1986)

[12] T S Hahm and Liu Chen, Phys. Fluids 291891 (1986)

[13] A H Glasser, J M Greene and J L Johnson, Phys. Fluids 18875 (1975)

[14] W M Tang, J W Connor and R J Hastie, Nucl. Fusion 201439 (1980)

[15] J W Connor, Plasma Physics 15765 (1973)

[16] J W Connor, R J Hastie and P Helander, 'Linear Tearing Mode Equations at Low Collisionality', UKAEA Report UKAEA FUS 550 (2008)

[17] J W Connor, R C Grimm, R J Hastie and P M Keeping, Nucl. Fusion 13211 (1973)

[18] M N Rosenbluth, P H Rutherford, J B Taylor, E A Frieman, and L M Kovrizhnykh, Plasma Physics and Controlled Nuclear Fusion Research, 1971 (IAEA Vienna, 1971), Vol. I, p.495

[19] S K Wong and V S Chan, Phys. Plasmas 12092513 (2005)

[20] A H Glasser, J M Greene and J L Johnson, Phys. Fluids 19567 (1976)

[21] J W Connor and R J Hastie, 'The effect of shaped cross sections on the ideal internal kink mode in a tokamak', Culham Report CLM-M 106, 1985 


\section{Appendix A: Some equilibrium relationships applied to the vorticity equation}

Let us first introduce the flux-surface-averaged quantities $\mathrm{E}, \mathrm{F}$ and $\mathrm{H}$ defined by Glasser et al. [13]

$$
\begin{aligned}
& \mathrm{E}=\frac{\mu_{0} \mathrm{p}_{0}^{\prime} \mathrm{q}}{\mathrm{q}^{\prime 2} \mathrm{I}\left\langle 1 / \mathrm{R}^{2}\right\rangle}\left[\frac{\mathrm{Iq}^{\prime}}{\left\langle\mathrm{B}^{2}\right\rangle}-\mathrm{V}^{\prime \prime}\right]\left\langle\frac{\mathrm{B}^{2}}{|\nabla \psi|^{2}}\right\rangle \\
& \mathrm{F}=\left(\frac{\mu_{0} \mathrm{p}_{0}^{\prime} \mathrm{q}}{\mathrm{q}^{\prime} \mathrm{I}\left\langle 1 / \mathrm{R}^{2}\right\rangle}\right)^{2}\left\{\mathrm{I}^{2}\left[\left\langle\frac{\mathrm{B}^{2}}{|\nabla \psi|^{2}}\right\rangle\left\langle\frac{1}{\mathrm{~B}^{2}|\nabla \psi|^{2}}\right\rangle-\left\langle\frac{1}{|\nabla \psi|^{2}}\right\rangle^{2}\right]+\left\langle\frac{1}{\mathrm{~B}^{2}}\right\rangle\left\langle\frac{\mathrm{B}^{2}}{|\nabla \psi|^{2}}\right\rangle\right\} \\
& \mathrm{H}=\frac{\mu_{0} \mathrm{p}_{0}^{\prime} \mathrm{q}}{\mathrm{q}^{\prime}\left\langle 1 / \mathrm{R}^{2}\right\rangle}\left[\left\langle\frac{1}{|\nabla \psi|^{2}}\right\rangle-\frac{\left\langle\frac{\mathrm{B}^{2}}{|\nabla \psi|^{2}}\right\rangle}{\left\langle\mathrm{B}^{2}\right\rangle}\right]
\end{aligned}
$$

where

$$
\mathbf{B}=\mathrm{I} \nabla \phi+\nabla \phi \times \nabla \psi, \text { so that } \mathbf{B} \cdot \nabla \theta=\frac{\mathrm{I}}{\mathrm{R}^{2} \mathrm{q}} \text { and }\langle\mathrm{A}\rangle \equiv \oint \frac{\mathrm{d} \theta}{\mathrm{B} \cdot \nabla \theta} \mathrm{A} / \oint \frac{\mathrm{d} \theta}{\mathrm{B} . \nabla \theta} .
$$

It is also convenient to introduce the quantity L defined in Refs. 2 and 5:

$$
\mathrm{L}=\frac{\mu_{0} \mathrm{p}_{0}^{\prime} \mathrm{q}}{\mathrm{q}^{\prime}\left\langle 1 / \mathrm{R}^{2}\right\rangle\left\langle\mathrm{B}^{2}\right\rangle}\left\langle\frac{\mathrm{B}^{2}}{|\nabla \psi|^{2}}\right\rangle
$$

We now consider separately the terms proportional to $\hat{\mathrm{p}}, \hat{\mathrm{p}}^{\prime}, \Psi$ and $\Psi^{\prime}$ in eqn. (88) when we recall eqn. (57):

$$
\tilde{\mathrm{p}}=\hat{\mathrm{p}}^{\prime}+\frac{\mathrm{n}}{\omega} \mathrm{p}_{0}^{\prime} \Psi
$$

First we evaluate the average curvature:

$$
\mathrm{K} \equiv\left\langle\frac{1}{\mathrm{~B}^{2}} \frac{\partial}{\partial \varphi}\left(\mu_{0} \mathrm{p}_{0}+\frac{\mathrm{B}^{2}}{2}\right)+\frac{\nabla \psi \cdot \nabla \theta}{\mathrm{R}^{2} \mathrm{~B}^{4}} \frac{\partial}{\partial \theta}\left(\frac{\mathrm{B}^{2}}{2}\right)\right\rangle
$$




$$
=\mu_{0} \mathrm{p}_{0}^{\prime}\left\langle\frac{1}{\mathrm{~B}^{2}}\right\rangle+\frac{1}{2}\left\langle\frac{1}{\mathrm{~B}^{2}} \frac{\partial}{\partial \psi} \mathrm{B}^{2}\right\rangle+\frac{1}{2}\left\langle\frac{1}{\mathrm{R}^{2} \mathrm{~B}^{2}} \frac{\partial}{\partial \theta}(\nabla \theta \cdot \nabla \psi)\right\rangle
$$

Now the Grad-Shafranov equation can be written (see eqn. (8) of Ref. 21, but in terms of our metric coefficients)

$$
\frac{\mathrm{I}}{\mathrm{R}^{2} \mathrm{q}}\left[\frac{\partial}{\partial \psi}\left(\frac{\mathrm{q}}{\mathrm{I}}|\nabla \psi|^{2}\right)+\frac{\mathrm{q}}{\mathrm{I}} \frac{\partial}{\partial \theta}(\nabla \psi \cdot \nabla \theta)\right]+\mu_{0} \mathrm{p}_{0}^{\prime}+\frac{\mathrm{II}^{\prime}}{\mathrm{R}^{2}}=0
$$

so that eqn. (A.4) becomes, after some algebra,

$$
\mathrm{K}=\frac{\mu_{0} \mathrm{p}_{0}^{\prime}}{2}\left\langle\frac{1}{\mathrm{~B}^{2}}\right\rangle+\frac{\mathrm{II}^{\prime}}{2}\left\langle\frac{1}{\mathrm{R}^{2} \mathrm{~B}^{2}}\right\rangle-\frac{\mathrm{q}^{\prime}}{2 \mathrm{q}}\left\langle\frac{|\nabla \psi|^{2}}{\mathrm{R}^{2} \mathrm{~B}^{2}}\right\rangle+\frac{\mathrm{I}^{\prime}}{2 \mathrm{I}}\left\langle\frac{|\nabla \psi|^{2}}{\mathrm{R}^{2} \mathrm{~B}^{2}}\right\rangle-\frac{1}{2} \frac{\partial}{\partial \psi}\left(\frac{\mathrm{IV}^{\prime}}{\mathrm{q}}\right)
$$

Finally, introducing $V^{\prime \prime}$, where $V^{\prime}=(q / I) \oint R^{2} d \theta$, we have

$$
\mathrm{K}=\frac{1}{2}\left[\mu_{0} \mathrm{p}_{0}^{\prime}\left\langle\frac{1}{\mathrm{~B}^{2}}\right\rangle+\frac{\mathrm{I}^{2} \mathrm{q}^{\prime}}{\mathrm{q}}\left\langle\frac{1}{\mathrm{R}^{2} \mathrm{~B}^{2}}\right\rangle-\frac{\mathrm{IV}^{\prime \prime}}{\mathrm{q}}\right]
$$

Then gathering terms in $\Psi$, we have a contribution $\mathrm{I}_{1} \Psi$ with

$$
\begin{gathered}
\mathrm{I}_{1}=\left(\mu_{0} \mathrm{p}_{0}^{\prime}\right)^{2}\left[\left\langle\frac{\mathrm{I}^{2}}{|\nabla \psi|^{2} \mathrm{~B}^{2}}\right\rangle\left\langle\frac{\mathrm{B}^{2}}{|\nabla \psi|^{2}}\right\rangle-\left\langle\frac{\mathrm{I}}{|\nabla \psi|^{2}}\right\rangle^{2}\right]+\frac{\mu_{0} \mathrm{p}_{0}^{\prime} \mathrm{q}^{\prime}}{\mathrm{q}}\left[\left\langle\frac{\mathrm{I}^{2}}{\mathrm{R}^{2}}\right\rangle\left\langle\frac{1}{|\nabla \psi|^{2}}\right\rangle-\left\langle\frac{\mathrm{B}^{2}}{|\nabla \psi|^{2}}\right\rangle\left\langle\frac{\mathrm{I}^{2}}{\mathrm{R}^{2} \mathrm{~B}^{2}}\right\rangle\right] \\
+2 \mu_{0} \mathrm{p}_{0}^{\prime}\left\langle\frac{1}{\mathrm{~B}^{2}} \frac{\partial}{\partial \psi}\left(\mu_{0} \mathrm{p}_{0}+\frac{\mathrm{B}^{2}}{2}\right)+\frac{\nabla \psi \cdot \nabla \theta}{\mathrm{R}^{2} \mathrm{~B}^{4}} \frac{\partial}{\partial \theta}\left(\frac{\mathrm{B}^{2}}{2}\right)\right\rangle\left\langle\frac{\mathrm{B}^{2}}{|\nabla \psi|^{2}}\right\rangle
\end{gathered}
$$

Using eqn. (A.8) and the definitions (A.1) we obtain

$$
\mathrm{I}_{1}=\frac{\mathrm{q}^{\prime 2}}{\mathrm{~V}^{\prime 2}}(\mathrm{E}+\mathrm{F}+\mathrm{H}) \equiv \frac{\mathrm{q}^{\prime 2}}{\mathrm{~V}^{\prime 2}}\left(\mathrm{D}_{\mathrm{I}}-\frac{1}{4}\right)
$$

Turning to the terms proportional to $\Psi^{\prime}$, we find these trivially vanish. For the terms in $\hat{\mathrm{p}}^{\prime}$, we find they readily combine to yield $\mathrm{I}_{2} \hat{\mathrm{p}}^{\prime}$, where 


$$
I_{2}=\frac{q^{\prime 2}}{V^{\prime 2}}(L+H)
$$

using the definition (A.2). Finally we consider the terms proportional to $\hat{\mathrm{p}}$; this calculation is identical to that of the terms in $\Psi$, leading to a contribution:

$I_{1} \hat{p}$. As a result, finally, we find eqn. (90) can be written in the form of eqn. (91).

\section{Appendix B: A summary of some symbols and notation employed}

Here we collect some of the definitions and symbols employed in this paper.

- Velocity space variables:

$\mathrm{v}, \lambda, \sigma$, and gyro-angle:

$$
\begin{aligned}
& \sigma=\mathrm{v}_{\|} /\left|\mathrm{v}_{\|}\right|: \lambda=\frac{2 \mu}{\mathrm{v}^{2}} ; \mathrm{v}_{\|}=\sigma \mathrm{v}(1-\lambda \mathrm{B})^{1 / 2} \\
& \mathrm{u}^{2}=\frac{\mathrm{mv}^{2}}{2 \mathrm{~T}}
\end{aligned}
$$

- Configuration space variables:

$\mathrm{x}, \theta$ and $\varphi$, a non-orthogonal set in which field line trajectories are straight $(\varphi=\mathrm{q} \theta)$.

$\mathrm{x}=\left(\psi-\psi_{0}\right)$ : the local flux variable. Prime is used to denote differential with respect to the poloidal flux, $\mathrm{x}$; thus

$\mathrm{h}_{\mathrm{j}}^{\prime}=\frac{\partial \mathrm{h}_{\mathrm{j}}}{\partial \mathrm{x}} ; \quad \hat{\mathrm{n}}_{\mathrm{j}}^{\prime}=\frac{\mathrm{d} \hat{\mathrm{n}}_{\mathrm{j}}}{\mathrm{dx}} ; \quad \hat{\mathrm{T}}_{\mathrm{j}}^{\prime}=\frac{\mathrm{d} \hat{\mathrm{T}}_{\mathrm{j}}}{\mathrm{dx}} ; \quad \hat{\mathrm{p}}_{\mathrm{j}}=\frac{\mathrm{d} \hat{\mathrm{p}}_{\mathrm{j}}}{\mathrm{dx}}$.

s, a normalised radial variable representing semi-collisional effects, see eqn. (41).

$$
\begin{aligned}
& \hat{\nabla}_{\|}=\frac{\mathrm{I}}{\mathrm{R}^{2} \mathrm{qB}} \frac{\partial}{\partial \theta} . \\
& \langle\mathrm{X}\rangle=\oint \mathrm{R}^{2} \mathrm{Xd} \theta / \oint \mathrm{R}^{2} \mathrm{~d} \theta .
\end{aligned}
$$


- Magnetic field variables

$\mathrm{q}$ is the safety factor; $\mathrm{R}$ the major radius, $\mathrm{I}=\mathrm{RB}_{\phi}$.

$\hat{\mathrm{s}}$ is the magnetic shear.

$$
\begin{aligned}
& \hat{\mathrm{V}}_{\|}=\frac{\sigma v \mathrm{~B}_{0}}{2} \int_{\lambda}^{\lambda_{\mathrm{c}}} \frac{\mathrm{d} \lambda^{\prime}}{\left\langle\left(1-\lambda^{\prime} \mathrm{B}\right)^{1 / 2}\right\rangle} \\
& \mathrm{f}_{\mathrm{c}}=\frac{3}{4} \mathrm{~B}_{0}^{2} \int_{0}^{\lambda_{\mathrm{c}}} \frac{\lambda \mathrm{d} \lambda}{\left\langle(1-\lambda \mathrm{B})^{1 / 2}\right\rangle} ; \quad \lambda_{\mathrm{c}}=\frac{1}{\mathrm{~B}_{\max }}
\end{aligned}
$$

$\mathrm{B}_{0}$ is an arbitrary constant magnetic field; a convenient choice is $\mathrm{B}_{\max }$, or $\left\langle\mathrm{B}^{2}\right\rangle^{1 / 2}$.

- Perturbations have the form $\mathrm{a}(\mathrm{x}) \exp \mathrm{i}(\mathrm{n} \varphi-\mathrm{m} \theta-\omega \mathrm{t})$

$\Psi=\frac{1}{i \omega} \hat{\nabla}_{\|} \mathrm{A}_{\|}$

$\mathrm{A}_{\|}$: longitudinal component of the perturbed vector potential.

$\Phi$ : perturbed electrostatic potential.

$\tilde{\mathrm{B}}_{\|}:$perturbed longitudinal magnetic field.

$\tilde{\mathrm{p}}$ : total perturbed pressure.

$\hat{\mathrm{n}}_{\mathrm{j}}, \hat{\mathrm{T}}_{\mathrm{j}}$ : contributions to density and temperature from the $\mathrm{h}_{\mathrm{j} 0}$ part of the perturbed distribution functions.

$\mathrm{u}_{\| \mathrm{j}}$ : longitudinal fluid velocity of species $\mathrm{j}$.

$\mathrm{u}_{\| j}^{*}$ : weighted parallel flow for species $\mathrm{j}$, as defined for electrons in eqn. (26)

and ions in eqn. (45).

$\Pi$ : radial momentum flux. 
- Frequencies

$$
\begin{aligned}
& \omega_{* j}^{\mathrm{T}}=\omega_{* j}\left[1+\eta_{\mathrm{j}}\left(\mathrm{u}_{\mathrm{j}}^{2}-\frac{3}{2}\right)\right], \quad \omega_{*_{j}}=-\mathrm{n} \frac{\mathrm{T}_{\mathrm{j}}}{\mathrm{e}_{\mathrm{j}}} \frac{\mathrm{d}\left(\ell \mathrm{nn} \mathrm{n}_{\mathrm{j}}\right)}{\partial \psi}, \\
& v_{\mathrm{ei}}=v_{0} / \mathrm{u}^{3}, v_{\mathrm{ee}}=v_{0} \phi(\mathrm{u}) / \mathrm{u}^{3} ; v_{0}=\frac{\sqrt{2} \mathrm{\pi n}_{\mathrm{e}} \mathrm{e}^{4} \ln \Lambda}{\mathrm{m}_{\mathrm{e}}^{1 / 2} \mathrm{~T}_{\mathrm{e}}^{3 / 2}} . \\
& v_{\mathrm{ii}}=v_{\mathrm{i}} \phi(\mathrm{u}) / \mathrm{u}^{3} ; v_{\mathrm{i}}=\frac{\sqrt{2} \mathrm{nn}_{\mathrm{e}} \mathrm{e}^{4} \ln \Lambda}{\mathrm{m}_{\mathrm{i}}^{1 / 2} \mathrm{~T}_{\mathrm{i}}^{3 / 2}} ; \phi(\mathrm{u})=\left(1-\frac{1}{2 \mathrm{u}^{2}}\right) \operatorname{erf}(\mathrm{u})+\frac{\mathrm{e}^{-\mathrm{u}^{2}}}{\mathrm{u} \sqrt{\pi}}, \\
& \text { so that } \tau_{\mathrm{i}}=\frac{3}{4 \sqrt{\pi}} \frac{1}{v_{\mathrm{i}}}, \quad \tau_{\mathrm{ei}}=\frac{3}{4 \sqrt{\pi}} \frac{1}{v_{0}} . \\
& \Omega_{\mathrm{j}}=\frac{\mathrm{e}_{\mathrm{j}} \mathrm{B}}{\mathrm{m}_{\mathrm{j}}} ; \quad \mathrm{e}_{\mathrm{e}}=-\mathrm{e}, \quad \mathrm{e}_{\mathrm{i}}=+\mathrm{e} .
\end{aligned}
$$

- Symbols used in obtaining vorticity equation

E, F, H, DI, L: symbols defined in Appendix A.

\section{- Equilibrium quantities}

$$
\begin{aligned}
& \Delta^{\prime} \text { - tearing mode stability parameter. } \\
& \tau=\mathrm{T}_{\mathrm{e}} / \mathrm{T}_{\mathrm{i}} \text {. } \\
& \mathrm{L}_{\mathrm{n}}, \quad \mathrm{L}_{\mathrm{T}}, \quad \mathrm{L}_{\mathrm{p}} \quad \text { density, temperature and pressure scale-lengths; } \\
& \eta_{\mathrm{j}}=\mathrm{d}\left(\ell \mathrm{nT}_{\mathrm{j}}\right) / \mathrm{d}\left(\ell \mathrm{nn}_{\mathrm{j}}\right)=\mathrm{L}_{\mathrm{n}_{\mathrm{j}}} / \mathrm{L}_{\mathrm{T}_{\mathrm{j}}} . \\
& \hat{\beta}, \hat{\gamma} \text { and } \mathrm{C} \text { normalised pressure, bootstrap current and collisionality parameters } \\
& \text { of the semi-collisional theory, see eqn. }(96) \text {. }
\end{aligned}
$$

- Semi-collisional neoclassical current

$\sigma_{\|}^{\mathrm{sc}}, \hat{\sigma}, \sigma_{\mathrm{n}}$ and $\sigma_{\mathrm{T}}$ semi-collisional conductivity eqn. (67), a normalised form in eqn. (96) and density gradient and temperature gradient contributions to eqn. (67). $\mathrm{D}=1+\mathrm{d}_{0} \mathrm{~s}^{2}+\mathrm{d}_{1} \mathrm{~s}^{4}$, denominator appearing in eqn. (40). 
$\lambda_{\mathrm{j}}, \mathrm{j}=1-4$ : coefficients of semi-collisionality effects defined in eqn. (40).

$\tilde{\mathrm{J}}:$ the bootstrap current factor in eqn. (64).

$\alpha_{n}, \alpha_{\mathrm{e}}$ and $\alpha_{\mathrm{i}}$ : coefficients of density and electron and ion temperature gradient contributions to bootstrap current, eqn. (64). 